\title{
HEAVY-ION ELEMENTAL ABUNDANCES IN LARGE SOLAR ENERGETIC PARTICLE EVENTS AND THEIR IMPLICATIONS FOR THE SEED POPULATION
}

\author{
M. I. DesaI \\ Southwest Research Institute, 6220 Culebra Road, San Antonio, TX 78238-5166 \\ G. M. Mason, R. E. Gold, and S. M. KRIMIGIS \\ Applied Physics Laboratory, The Johns Hopkins University, Laurel, MD 20723 \\ C. M. S. Cohen and R. A. Mewaldt \\ California Institute of Technology, Pasadena, CA 91125 \\ J. E. MAZUR \\ The Aerospace Corporation, Space Sciences Department-Chantilly; \\ 15049 Conference Center Drive, CH3/210 Chantilly, VA 20151 \\ AND \\ J. R. DWyer \\ Florida Institute of Technology, Melbourne, FL 32901 \\ Received 2006 February 13; accepted 2006 May 4
}

\begin{abstract}
We have surveyed the $\sim 0.1-10 \mathrm{MeV}$ nucleon ${ }^{-1}$ abundances of heavy ions from ${ }^{3} \mathrm{He}$ through $\mathrm{Fe}$ in 64 large solar energetic particle (LSEP) events observed on board the Advanced Composition Explorer from 1997 November through 2005 January. Our main results are (1) the $0.5-2.0 \mathrm{MeV}$ nucleon ${ }^{-1}{ }^{3} \mathrm{He} /{ }^{4} \mathrm{He}$ ratio is enhanced between factors of $\sim 2-$ 150 over the solar wind value in $29(\sim 46 \%)$ events. (2) The Fe/O ratio in most LSEP events decreases with increasing energy up to $\sim 60 \mathrm{MeV}$ nucleon ${ }^{-1}$. (3) The $\mathrm{Fe} / \mathrm{O}$ ratio is independent of CME speed, flare longitude, event size, the ${ }^{3} \mathrm{He} /{ }^{4} \mathrm{He}$ ratio, the pre-event $\mathrm{Fe} / \mathrm{O}$ ratio, and solar activity. (4) The LSEP abundances exhibit unsystematic behavior as a function of $M / Q$ ratio when compared with average solar wind values. (5) The survey-averaged abundances are enhanced with increasing $M / Q$ ratio when compared with quiet coronal values and with average gradual SEP abundances obtained at 5-12 MeV nucleon ${ }^{-1}$. (6) The event-to-event variations in LSEP events are remarkably similar to those seen in CME-driven IP shocks and in ${ }^{3} \mathrm{He}$-rich SEP events. The above results cannot be explained by simply invoking the current paradigm for large gradual SEP events, i.e., that CME-driven shocks accelerate a seed population dominated by ambient coronal or solar wind ions. Instead, we suggest that the systematic $M / Q$-dependent enhancements in LSEP events are an inherent property of a highly variable suprathermal seed population, most of which is accelerated by mechanisms that produce heavy-ion abundances similar to those observed in impulsive SEP events. This heavy-ion-enriched material is subsequently accelerated at CME-driven shocks near the Sun by processes in which ions with higher $M / Q$ ratios are accelerated less efficiently, thus causing the $\mathrm{Fe} / \mathrm{O}$ ratios to decrease with increasing energy.
\end{abstract}

Subject heading: Sun: particle emission

Online material: color figure

\section{INTRODUCTION}

The earliest observations of solar energetic particle (SEP) events extending up to $\mathrm{GeV}$ energies were made with ionization chambers and neutron monitors (Forbush 1946; Meyer et al. 1956). Since such events, also known as ground level events, were associated with the maximum of $\mathrm{H} \alpha$ flares on the Sun, it was presumed that there was a causal relationship between the flare and the energetic particles observed at $1 \mathrm{AU}$. However, on the basis of a close association between the SEP events and slow-drifting type II and various kinds of type IV radio bursts, Wild et al. (1963) proposed that the energetic particles might be accelerated at magnetohydrodynamic shock waves that typically accompanied the flares. Later, associations between "pure" electron events and flares that only exhibited metric type III emission on the one hand, and between "mixed" events with protons and relativistic electrons and flares with type II/IV radio events on the other hand, led Lin (1970) to propose a "two-phase" acceleration process for the SEP events observed in interplanetary (IP) space.
Despite these results, a two-class paradigm for SEP events was not generally accepted until the mid-1990s. The close association between coronal mass ejections (CMEs) observed on Skylab and large solar proton events (SPEs) led Kahler et al. (1978) to suggest an important role for the CME either in creating open field lines for flare particles to escape into the IP medium or for the protons to be accelerated near a region above or around the outward moving ejecta far above the flare site. Subsequently, detailed analyses of flare durations, longitudinal distributions from multispacecraft observations, ionic charge state and elemental composition measurements, and clearer associations with radio bursts led to the prevailing viewpoint that SEP events observed near 1 AU belong to two distinct classes, namely, "impulsive" and "gradual" (e.g., Cliver et al. 1982; Kocharov 1983; Kahler et al. 1984; Luhn et al. 1984; Mason et al. 1984; Cane et al. 1986; Reames 1988).

In the two-class paradigm, the gradual events occur as a result of diffusive acceleration at CME-driven coronal and IP shocks, while the impulsive events are attributed to stochastic acceleration 
during magnetic reconnection in solar flares (e.g., Reames 1999). The gradual or CME-related events typically last several days and have larger fluences, while the impulsive or flare-related events typically last a few hours and have smaller fluences. Impulsive events are typically confined to field lines originating in a narrow range of longitudes in the western solar hemisphere near the flare site. In contrast, the ions in gradual events are accelerated by expanding large-scale CME-driven shocks and therefore populate magnetic field lines over a significantly broad range of solar longitudes (Cane et al. 1988).

The distinction between impulsive and gradual SEP events also includes differences in the energetic particle composition measurements above $\sim 1 \mathrm{MeV}$ nucleon ${ }^{-1}$ and radio burst properties (e.g., Cane et al. 1986). In particular, the flare-related impulsive SEP events are electron-rich and associated with type III radio bursts. These events also have ${ }^{3} \mathrm{He} /{ }^{4} \mathrm{He}$ ratios enhanced between factors of $10^{3}$ and $10^{4}, \mathrm{Ne}-\mathrm{Si}$ abundances enhanced between factors of $\sim 2$ and 4 , and $\mathrm{Fe} / \mathrm{O}$ ratios enhanced by up to a factor of 10 over the corresponding solar wind values (Reames 1988). The impulsive SEP events also have mean ionization states for Fe of about $\sim 20$, which was interpreted as being indicative of temperatures of $\sim 10 \mathrm{MK}$ in the source region (Klecker et al. 1984; Luhn et al. 1987). In contrast, the gradual events are proton-rich, are associated with type II bursts, and have average $\mathrm{Fe} / \mathrm{O}$ ratios of $\sim 0.1$ with mean Fe ionization states of $\sim 10-14$ (e.g., Luhn et al. 1984; Reames 1999; Cliver 2000). The Fe charge state measurements in LSEP events were interpreted as being indicative of source temperatures of about $\sim 1.5-2 \mathrm{MK}$ (e.g., Gloeckler et al. 1976; Hovestadt et al. 1981; Luhn et al. 1984), which is typical of the ambient corona and the solar wind. Finally, although reports of large gradual SEP events with significant ${ }^{3} \mathrm{He}$ did exist (Garrard et al. 1973; Dietrich 1973; Van Hollebeke et al. 1990), such events were rare and were interpreted as being "mixed." In general, the LSEP events were assumed to have ${ }^{3} \mathrm{He} /{ }^{4} \mathrm{He}$ ratios similar to that measured in the solar wind, primarily because instruments flown up until the mid-1990s could not separate the ${ }^{3} \mathrm{He}$ nuclei from the more abundant ${ }^{4} \mathrm{He}$ nuclei when the ${ }^{3} \mathrm{He} /{ }^{4} \mathrm{He}$ ratio fell below the $10 \%$ level (e.g., see review by Reames 1999 and references therein).

These earlier surveys also showed that the heavy-ion abundances in individual gradual events were systematically enhanced or depleted with increasing $M / Q$ values when compared with the average gradual SEP abundances measured above a few $\mathrm{MeV}$ nucleon $^{-1}$ (e.g., Breneman \& Stone 1985; Meyer 1985), which, in turn, were found to be roughly similar to the spectroscopic abundances in the quiet solar corona (e.g., Sheeley 1996). Such $M / Q$-dependent fractionation was attributed primarily to the effects of rigidity-dependent acceleration and transport processes acting on a seed population composed predominantly of ambient coronal material (e.g., Breneman \& Stone 1985).

Until the early 1980s, the IP shocks observed near Earth were also believed to be produced by solar flares (e.g., Rust 1987; Dryer 1987; Bone 1992). Such IP shocks are often accompanied by enhancements in the intensities of energetic ions near $1 \mathrm{AU}-$ the so-called energetic storm particle (ESP) events (e.g., Bryant et al. 1962). Later, however, it became clear that the IP shocks are driven by fast CMEs propagating through IP space (e.g., Gosling 1993). Together with the composition measurements available at the time, these findings led to the viewpoint (e.g., Reames 1999) that a CME-driven shock accelerates ambient coronal or solar wind plasma near the Sun and in IP space and produces a LSEP event while the same shock accelerates solar wind ions en route to Earth and produces an ESP event (e.g., Lee 1983; Forman \& Webb 1985).
Some researchers showed evidence that the source material for ESP events could originate from the suprathermal tail of the solar wind (Gosling et al. 1981) or comprise suprathermal ions accelerated during the accompanying solar flares (e.g., Tsurutani \& Lin 1985; Tan et al. 1989). However, such ideas regarding alternative source populations were not widely accepted because of the following reasons. (1) Early theoretical studies based on diffusive shock acceleration theory (e.g., Lee 1983) were reasonably successful in predicting many features of some ESP events (e.g., Kennel et al. 1986). (2) Instruments flown during the 1970s and 1980s lacked the energy coverage, sensitivity, and resolution to measure small differences between solar wind and energetic particle abundances.

NASA's Advanced Composition Explorer ( $A C E$ ) spacecraft, launched in 1997 August (Stone et al. 1998a) to orbit around the sunward Lagrangian point, was designed to explore questions regarding the origin of the source populations with the aid of several high-resolution and high-sensitivity mass spectrometers. Indeed, results from $A C E$ have shown a remarkable degree of variability in the composition and energy spectra during LSEP events (e.g., Cohen et al. 1999, 2000; Mason et al. 1999b; Möbius et al. 2000; Tylka et al. 2005), in ESP events (Desai et al. 2001, 2003, 2004), and in ${ }^{3} \mathrm{He}$-rich SEP events (Mason et al. 2002, 2004; Slocum et al. 2003; Wiedenbeck et al. 2003). In particular, Mason et al. (1999a) found that the $\sim 1 \mathrm{MeV}$ nucleon ${ }^{-1}{ }^{3} \mathrm{He} /{ }^{4} \mathrm{He}$ ratio in a sizable fraction of LSEP events is enhanced between factors of $\sim 5-140$ over the slow solar wind value of $\sim 4.05 \times 10^{-4}$ (Gloeckler \& Geiss 1998). Such ${ }^{3} \mathrm{He}$ enrichments were also found above $\sim 5 \mathrm{MeV}$ nucleon $^{-1}$ (Cohen et al. 1999, 2000; Wiedenbeck et al. 2000; Torsti et al. 2002). In addition, the Fe/O ratios and mean ionization states of $\mathrm{Fe}$ and other heavy ions in many LSEP events were found to increase with increasing energy (e.g., Möbius et al. 1999, 2000; Mazur et al. 1999; Popecki et al. 2002; Cohen et al. 2003; Leske et al. 2003; Labrador et al. 2003; Tylka et al. 2005). Finally, the average Fe/O ratios in several LSEP events were substantially enhanced relative to the coronal values (Cohen et al. 1999, 2003; Cane et al. 2003; Tylka et al. 2005; Mewaldt et al. 2006). Thus, these new results posed serious challenges for the notion that LSEP events are caused by the acceleration of ambient coronal or solar wind ions at CME-driven shocks (e.g., Mason et al. 1999a).

Indeed, the presence of residual ${ }^{3} \mathrm{He}$ ions from prior impulsive flares during a substantial fraction of the time in IP space near 1 AU led Mason et al. (1999a) to suggest that CME-driven shocks might occasionally encounter and reaccelerate suprathermal flareassociated material that was enriched in ${ }^{3} \mathrm{He}$ and heavy ions (see also Wiedenbeck et al. 2003), thereby causing the unusual compositional characteristics in some LSEP events. The idea that CME-driven shocks can reaccelerate suprathermal material from prior SEP events has subsequently been explored in a number of studies of odd compositional and spectral behavior in IP shock events (e.g., Desai et al. 2001, 2003, 2004; Channok et al. 2005) and in LSEP events (Tylka et al. 2001, 2005; Kocharov \& Torsti 2003; Li \& Zank 2005).

In this paper we extend the earlier work of Mason et al. (1999a) and survey the heavy-ion abundances from ${ }^{3} \mathrm{He}$ to $\mathrm{Fe}$ in the $\sim 0.1-10 \mathrm{MeV}$ nucleon ${ }^{-1}$ energy range during 64 LSEP events of cycle 23 . In order to identify the source populations for LSEP events, we compare the heavy-ion abundances with those measured in the fast and slow solar wind (von Steiger et al. 2000) and in particle populations accelerated near the Sun (Reames 1995a; Mason et al. 2004) and in IP space (Desai et al. 2003). We also examine the event-to-event enhancement pattern of the heavyion abundances and discuss our results in terms of fractionation 
effects due to rigidity-dependent acceleration and transport to $1 \mathrm{AU}$ and due to the properties of the seed populations.

Contrary to previous surveys above $\sim 1 \mathrm{MeV}$ nucleon $^{-1}$, we find evidence for the occurrence of two distinct but opposite $M / Q$-dependent fractionation processes in LSEP events. On the one hand, the heavy-ion abundances exhibit event-to-event $M / Q$ dependent enhancements relative to ambient coronal values that are remarkably similar to those seen in IP shock events (Desai et al. 2004) and ${ }^{3} \mathrm{He}$-rich SEP events (Mason et al. 2004). On the other hand, the $\mathrm{Fe} / \mathrm{O}$ ratio in most LSEP events decreases with increasing energy in the $\sim 0.1-60 \mathrm{MeV}$ nucleon ${ }^{-1}$ energy range, indicative of mechanisms in which ions with higher $M / Q$ ratios are accelerated less efficiently. We suggest that the $M / Q$ dependent enhancements in LSEP events do not occur due to acceleration and transport processes, as previously believed (e.g., Breneman \& Stone 1985; Meyer 1985). Rather, they are an inherent property of a suprathermal seed population that is primarily produced by processes that may be similar to those occurring during impulsive SEP events (e.g., Mason et al. 2004). This heavyion-enriched material is subsequently accelerated at CME-driven shocks by rigidity-dependent processes similar to those occurring during IP shock events (see Desai et al. 2004), thus causing the energy spectra to exhibit species-dependent breaks between $\sim 1$ and $10 \mathrm{MeV}$ nucleon ${ }^{-1}$ (e.g., Mewaldt et al. 2005b, 2005a; Cohen et al. 2005) and the $\mathrm{Fe} / \mathrm{O}$ ratios to decrease with increasing energy.

\section{INSTRUMENTATION AND DATA ANALYSIS}

\subsection{Instrumentation}

We use energetic particle measurements between $\sim 0.1$ and a few $\mathrm{MeV}$ nucleon $^{-1}$ obtained by the Ultra-Low Energy Isotope Spectrometer (ULEIS), which is a time-of-flight mass spectrometer with a geometry factor of $\sim 1 \mathrm{~cm}^{2} \mathrm{sr}$ and a $50 \mathrm{~cm}$ flight path (Mason et al. 1998). Supplementary measurements between $\sim 5$ and $60 \mathrm{MeV}$ nucleon ${ }^{-1}$ are obtained by the Solar Isotope Spectrometer (SIS), which is a multidetector $d E / d x$ versus residual energy spectrometer with two telescopes and a geometry factor of $\sim 38 \mathrm{~cm}^{2} \mathrm{sr}$ (Stone et al. 1998b). Both sensors were designed to achieve sensitivity and mass resolution that exceeded those of previous instruments in similar energy ranges.

\subsection{Event Selection}

We started with a list of 85 SPEs that affected Earth's environment between 1997 November and 2005 January. These events were identified from integral 5 minute averaged proton fluxes measured by the National Oceanic and Atmospheric Administration (NOAA) Geostationary Operational Environmental Satellite (GOES) spacecraft at geosynchronous orbit. ${ }^{1}$ The start of a given SPE is identified when three consecutive data points with proton flux for energies $>10 \mathrm{MeV}$ exceeds 10 particle flux units $\left(1 \mathrm{pfu}=1\right.$ proton $\left.\mathrm{cm}^{-2} \mathrm{sr}^{-1} \mathrm{~s}^{-1}\right)$.

The above SPE list does not make any distinction between particles accelerated near the Sun and those associated with CME-driven IP shocks near 1 AU. However, the ion intensities measured at $A C E$ during many SPE intervals were dominated by ESP events (see Desai et al. 2004). In order to measure ions that are accelerated predominantly during the SEP event, we excluded 15 events in which the SPE time intervals were affected by the following two factors: (1) ULEIS intensities were completely dominated by ESP events, and/or (2) the SPE occurred during complex time periods such that the ULEIS intensities included

\footnotetext{
1 The list is maintained at http://solar.sec.noaa.gov/ftpdir/indices/SPE.txt.
}

contributions from multiple SEP and ESP events. In addition, ULEIS did not measure any significant increases in the $\mathrm{O}$ intensities during five NOAA SPEs. Finally, no data were available for the 1998 November 14 08:10 SPE, as ULEIS was switched off during the Leonid meteor shower. A complete list of the excluded 21 events is provided at the bottom of Table 1 .

\subsection{Selection of Sampling Intervals}

The above analysis left us with a total of 64 events listed in Table 1. The properties of the associated CME and the flare are also provided. Events during which $A C E$ is reasonably well connected to the acceleration site near the Sun often exhibit normal velocity dispersion (e.g., see Mazur et al. 2000). In such cases, it is necessary to account for the fact that the higher energy ions often arrive within an hour of the launch of the CME or the onset of the associated flare, while ions below $\sim 0.5 \mathrm{MeV}$ nucleon $^{-1}$ arrive several hours later.

To illustrate the procedure for selecting energy-dependent sampling intervals for LSEP events, we show two examples of our observations in Figures 1 and 2. In each figure, panel $(a)$ displays the time intensity profiles of $\sim 0.15-50 \mathrm{MeV}^{\text {nucleon }}{ }^{-1}$ $\mathrm{O}$ ions measured by ULEIS and SIS, while panel $(b)$ shows a two-dimensional spectrogram of the expected arrival times for ions traveling along a 1.2 AU path length versus the measured arrival time of $0.04-10 \mathrm{MeV}$ nucleon ${ }^{-1} \mathrm{C}-\mathrm{Fe}$ ions. The dashed white lines show the expected velocity dispersion for ions traveling along a 1.2 AU Archimedian spiral path length from the Sun.

Figure 1 shows that from 23:20 UT on 2001 November 22, the $\mathrm{O}$ intensities above $\sim 1 \mathrm{MeV}$ nucleon ${ }^{-1}$ increased dramatically and exhibited velocity dispersion during the onset of event 37, which was associated with the flare and halo CME from AR 9704 at S17W24 as listed in Table 1. Figure 2 shows similar dispersion effects at the start of event 49 at 01:40 UT on 2002 August 24, which was associated with an X3 flare and a CME from AR 10069 at S02W81. In essence, both figures show that the $\mathrm{O}$ intensities exhibit velocity dispersion during the onsets and that a common sampling interval for all energies will also include contributions from the decay of prior events at the lower energies.

In order to exclude preexisting ion populations and take account of the observed velocity dispersion during the events, we first identified a single time interval (yellow shaded portions) starting with the onset of the associated flare and ending somewhat subjectively with the occurrence of either of the following: (1) the $\sim 1 \mathrm{MeV}$ nucleon ${ }^{-1} \mathrm{O}$ intensity decays completely (see Fig. 1), or (2) ULEIS starts sampling ions associated either with an IP shock (see Fig. 2) or another SEP event. The energydependent sampling intervals were then identified from the expected travel time versus arrival time plots as shown by the trapezoids in the bottom panels. Note, however, that the ion intensities measured by ULEIS and SIS during the event in Figure 1 are dominated by an ESP event (also see Desai et al. 2004). In order to measure the heavy-ion abundances accelerated near the Sun in such events, we identified and excluded the ESP portion (orange shaded region) from the sampling interval. For some intense events, the excluded ESP time intervals also included periods when ULEIS suffered loss in efficiency or from saturation effects (see Desai et al. 2003).

\subsection{Calculation of ${ }^{3} \mathrm{He} /{ }^{4} \mathrm{He}$ Ratio}

Table 2 lists the sampling intervals for $\sim 1 \mathrm{MeV}$ nucleon $^{-1}$ ions along with the heavy-ion abundances for each of the 64 events in our survey. The ${ }^{3} \mathrm{He} /{ }^{4} \mathrm{He}$ ratios are calculated by forming a 
TABLE 1

LSEP Events in This Survey

\begin{tabular}{|c|c|c|c|c|c|c|c|c|c|c|}
\hline \multicolumn{5}{|c|}{ NOAA GOES Particle Event } & \multicolumn{6}{|c|}{ Associated CME, Flare, and Active Region } \\
\hline SEP & Year & $\begin{array}{c}\text { Start Time } \\
\text { (month day/UT) }\end{array}$ & $\begin{array}{c}\text { Peak Time } \\
\text { (month day/UT) }\end{array}$ & $\begin{array}{l}\text { Peak Proton Flux } \\
(\text { pfu at }>10 \mathrm{MeV})\end{array}$ & $\begin{array}{l}\text { CME Location/ } \\
\text { Day-Time }\end{array}$ & $\begin{array}{l}\text { CME Speed } \\
\quad\left(\mathrm{km} \mathrm{s}^{-1}\right)\end{array}$ & $\begin{array}{c}\text { Maximum } \\
\text { (month day/UT) }\end{array}$ & Importance & Location & NOAA AR \\
\hline $1 \ldots$ & 1997 & Nov 04/08:30 & Nov 04/11:20 & 72 & $\mathrm{~W} / 040610$ & 785 & Nov 04/05:58 & $\mathrm{X} 2 / 2 \mathrm{~B}$ & S14W33 & 8100 \\
\hline $2 .$. & 1998 & Apr 20/14:00 & Apr 21/12:05 & 1700 & W/20 1007 & 1863 & Apr 20/10:21 & M1/EPL & S43W90 & 8194 \\
\hline $3 \ldots$ & 1998 & May 06/08:45 & May 06/09:45 & 210 & W/06 0829 & 1099 & May 06/08:09 & $\mathrm{X} 2 / 1 \mathrm{~N}$ & S11W65 & 8210 \\
\hline $4 \ldots \ldots \ldots \ldots$ & 1998 & Aug 24/23:55 & Aug 26/10:55 & 670 & $\ldots$ & $\ldots$ & Aug 24/22:12 & $\mathrm{X} 1 / 3 \mathrm{~B}$ & N30E07 & 8307 \\
\hline $5 \ldots \ldots \ldots \ldots$ & 1998 & Sep 25/00:10 & Sep 25/01:30 & 44 & $\ldots$ & $\ldots$ & Sep $23 / 07: 13$ & $\mathrm{M} 7 / 3 \mathrm{~B}$ & N18E09 & 8340 \\
\hline $6 \ldots \ldots \ldots \ldots$ & 1998 & Sep 30/15:20 & Oct $01 / 00: 25$ & 1200 & $\ldots$ & $\ldots$ & Sep 30/13:50 & $\mathrm{M} 2 / 2 \mathrm{~N}$ & N23W81 & 8340 \\
\hline $7 \ldots \ldots \ldots \ldots$ & 1998 & Nov $08 / 02: 45$ & Nov 08/03:00 & 11 & $\mathrm{~W} / 072054$ & 750 & & $\ldots$ & & $\ldots$ \\
\hline $8 \ldots \ldots \ldots \ldots$ & 1999 & Jan 23/11:05 & Jan 23/11:35 & 14 & . & $\ldots$ & Jan 20/20:05 & M5 & N27E90 & $\ldots$ \\
\hline 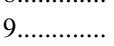 & 1999 & Apr 24/18:04 & Apr 25/00:55 & 32 & Halo/24 1331 & 1495 & & $\ldots$ & NW150 ${ }^{\mathrm{b}}$ & $\cdots$ \\
\hline $10 \ldots \ldots \ldots$ & 1999 & May 05/18:20 & May 05/19:55 & 14 & Halo/03 0606 & 1584 & May 3/06:02 & $\mathrm{M} 4 / \mathrm{N} 2$ & N15E32 & 8525 \\
\hline $11 \ldots \ldots \ldots . . .$. & 1999 & Jun 02/02:45 & Jun 02/10:10 & 48 & Halo/01 1937 & 1772 & Jun 01/19:30 & $\ldots$ & $\mathrm{N} \mathrm{W} 120^{\mathrm{b}}$ & $\ldots$ \\
\hline $12 \ldots \ldots \ldots$ & 1999 & Jun 04/09:25 & Jun 04/10:55 & 64 & NW/04 0726 & 2230 & Jun 04/07:03 & $\mathrm{M} 3 / 2 \mathrm{~B}$ & N17W69 & 8552 \\
\hline $13 \ldots \ldots \ldots$ & 2000 & Apr 04/20:55 & Apr 05/09:30 & 55 & W/04 1632 & 1188 & Apr 04/15:41 & $\mathrm{C} 9 / 2 \mathrm{~F}$ & N16W66 & 8933 \\
\hline $14 \ldots \ldots \ldots \ldots$ & 2000 & Jun $07 / 13: 35$ & Jun 08/09:40 & 84 & Halo/06 1554 & 1119 & Jun $06 / 15: 25$ & $\mathrm{X} 2 / 3 \mathrm{~B}$ & $\mathrm{~N} 33 \mathrm{E} 25^{\mathrm{c}}$ & 9026 \\
\hline $15 \ldots \ldots \ldots$ & 2000 & Jun 10/18:05 & Jun 10/20:45 & 46 & Halo/10 1708 & 1108 & Jun 10/17:02 & $\mathrm{M} 5 / 3 \mathrm{~B}$ & N22W38 & 9026 \\
\hline $16 \ldots \ldots \ldots$ & 2000 & Jul 14/10:45 & Jul 15/12:30 & 24000 & Halo/14 1054 & 1674 & Jul 14/10:24 & $\mathrm{X} 5 / 3 \mathrm{~B}$ & N22W07 & 9077 \\
\hline $17 \ldots \ldots \ldots$. & 2000 & Jul 22/13:20 & Jul 22/14:05 & 17 & NW/22 1154 & 1230 & Jul 22/11:34 & $\mathrm{M} 3 / 2 \mathrm{~N}$ & N14W56 & 9085 \\
\hline $18 \ldots \ldots \ldots . . .$. & 2000 & Sep 12/15:55 & Sep 13/03:40 & 320 & Halo/12 1154 & 1550 & Sep $12 / 12: 13$ & $\mathrm{M} 1 / 2 \mathrm{~N}$ & S17W09 & $\ldots$ \\
\hline $19 \ldots \ldots \ldots$ & 2000 & Oct $16 / 11: 25$ & Oct $16 / 18: 40$ & 15 & Halo/16 0727 & 1336 & Oct $16 / 07: 28$ & M2 & N04W90 & 9182 \\
\hline $20 \ldots \ldots \ldots$ & 2000 & Oct $26 / 00: 40$ & Oct 26/03:40 & 15 & $\mathrm{Halo} / 250826$ & 770 & Oct $25 / 11: 25$ & $\mathrm{C} 4$ & $\mathrm{~W} 50^{\mathrm{c}}$ & 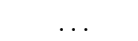 \\
\hline $21 \ldots \ldots \ldots . .$. & 2000 & Nov $08 / 23: 50$ & Nov 09/16:00 & 14800 & Halo/08 2306 & $1738^{\mathrm{c}}$ & Nov $08 / 23: 28$ & M7/multiple & $\mathrm{N} 10 \mathrm{~W} 77^{\mathrm{c}}$ & 9212 \\
\hline $22 \ldots \ldots \ldots \ldots$ & 2000 & Nov 24/15:20 & Nov 26/20:30 & 942 & Halo/24 0530 & 994 & Nov 24/05:02 & $\mathrm{X} 2 / 3 \mathrm{~B}$ & N20W05 & 9236 \\
\hline $23 \ldots \ldots \ldots \ldots$ & 2001 & Jan 28/20:25 & Jan 29/06:55 & 49 & Halo/28 1554 & 916 & Jan 28/16:00 & $\mathrm{M} 1 / 1 \mathrm{~N}$ & S04W59 & 9313 \\
\hline $24 \ldots$ & 2001 & Apr 02/23:40 & Apr 03/07:45 & 1110 & NW/02 2206 & 2505 & Apr 02/21:51 & $\mathrm{X} 20$ & $\mathrm{~N} 19 \mathrm{~W} 72^{\mathrm{c}}$ & 9393 \\
\hline $25 \ldots$. & 2001 & Apr 10/08:50 & Apr 11/20:55 & 355 & Halo/10 0530 & 2411 & Apr 10/05:26 & $\mathrm{X} 2 / 3 \mathrm{~B}$ & S23W09 & 9415 \\
\hline $26 \ldots \ldots \ldots . .$. & 2001 & Apr 15/14:10 & Apr 15/19:20 & 951 & W/15 1406 & 1199 & Apr 15/13:50 & $\mathrm{X} 14 / 2 \mathrm{~B}$ & S20W85 & 9415 \\
\hline $27 \ldots \ldots \ldots . .$. & 2001 & Apr 18/03:15 & Apr 18/10:45 & 321 & $\mathrm{SW} / 180230$ & 2465 & Apr 18/02:14 & $\mathrm{C} 2 / 2 \mathrm{~B}$ & $\mathrm{~S} 20 \mathrm{~W} 120^{\mathrm{b}}$ & 9415 \\
\hline $28 \ldots \ldots \ldots . .$. & 2001 & Apr 28/04:30 & Apr 28/05:00 & 57 & Halo/26 1230 & 1006 & Apr 26/13:12 & $\mathrm{M} 7 / 2 \mathrm{~B}$ & N17W31 & 9433 \\
\hline $29 \ldots \ldots \ldots$ & 2001 & May 07/19:15 & May 08/07:55 & 30 & NW/07 1206 & 1223 & May 07/12:20 & $\mathrm{C} 3 / \mathrm{SF}$ & $\mathrm{N} 26 \mathrm{~W} 32^{\mathrm{c}}$ & 9445 \\
\hline $30 \ldots \ldots \ldots$ & 2001 & June $15 / 17: 50$ & June 16/00:05 & 26 & $\mathrm{~W} / 151648$ & 1701 & & & $\mathrm{~S}$ W130 & $\ldots$ \\
\hline $31 \ldots \ldots$ & 2001 & Aug 10/10:20 & Aug 10/11:55 & 17 & W/9 2354 & 909 & Aug 09/18:34 & $\mathrm{C} 7 / 1 \mathrm{~F}$ & $\mathrm{~S} 30 \mathrm{E} 90^{\mathrm{c}}$ & 9570 \\
\hline $32 \ldots \ldots \ldots \ldots$ & 2001 & Aug 16/01:35 & Aug 16/03:55 & 493 & Halo/15 2354 & 1575 & $\ldots$ & $\ldots$ & $\mathrm{W} 140 ?^{\mathrm{b}}$ & $\ldots$ \\
\hline $33 \ldots \ldots \ldots . .$. & 2001 & Sep $24 / 12: 15$ & Sep $25 / 22: 35$ & 12900 & Halo/24 1030 & 2402 & Sep $24 / 10: 38$ & $\mathrm{X} 2 / 2 \mathrm{~B}$ & S16E23 & 9632 \\
\hline $34 \ldots \ldots \ldots \ldots$ & 2001 & Oct $01 / 11: 45$ & Oct $02 / 08: 10$ & 2360 & $\mathrm{SW} / 010530$ & 1405 & Oct $01 / 05: 15$ & M9 & $\mathrm{S} 18 \mathrm{~W} 80^{\mathrm{c}}$ & 9628 \\
\hline $35 \ldots \ldots \ldots$. & 2001 & Oct $19 / 22: 25$ & Oct 19/22:35 & 11 & Halo/19 1650 & 901 & Oct $19 / 16: 30$ & $\mathrm{X} 1 / 2 \mathrm{~B}$ & N15W29 & 9661 \\
\hline $36 \ldots \ldots \ldots . .$. & 2001 & Nov $04 / 17: 05$ & Nov $06 / 02: 15$ & 31700 & Halo/04 1635 & 1810 & Nov 04/16:20 & $\mathrm{X} 1 / 3 \mathrm{~B}$ & N06W18 & 9684 \\
\hline $37 \ldots \ldots \ldots \ldots$ & 2001 & Nov 22/23:20 & Nov $24 / 05: 55$ & 18900 & Halo/22 2330 & 1437 & Nov $22 / 23: 30$ & $\mathrm{M} 9 / 2 \mathrm{~N}$ & $\mathrm{~S} 17 \mathrm{~W} 24^{\mathrm{c}}$ & 9704 \\
\hline $38 \ldots \ldots \ldots \ldots$ & 2001 & Dec 26/06:05 & Dec 26/11:15 & 779 & $\mathrm{~W} / 260530$ & $1446^{\mathrm{c}}$ & Dec 26/05:40 & $\mathrm{M} 7 / 1 \mathrm{~B}$ & N08W54 & 9742 \\
\hline $39 \ldots \ldots \ldots$ & 2001 & Dec 30/02:45 & Dec 31/16:20 & 108 & Halo/30 1230 & 718 & $\ldots$ & $\ldots$ & $\ldots$ & $\ldots$ \\
\hline $40^{\mathrm{d}} \ldots \ldots \ldots$ & 2002 & Jan 10/20:45 & Jan 11/05:30 & 91 & E/10 1331 & 444 & Jan $10 / 20: 45$ & $\mathrm{C} 2$ & N14W10 & 9773 \\
\hline $41 \ldots \ldots \ldots . .$. & 2002 & Feb 20/07:30 & Feb 20/07:55 & 13 & $\mathrm{~W} / 200630$ & 952 & Feb 20/06:12 & $\mathrm{M} 5 / 1 \mathrm{~N}$ & N12W72 & 9825 \\
\hline $42 \ldots \ldots \ldots . .$. & 2002 & Mar 22/20:20 & Mar 23/13:20 & 16 & Halo/22 1106 & 1750 & Mar 22/11:14 & M1 & S W90 & 9866 \\
\hline $43 \ldots \ldots \ldots$ & 2002 & Apr 21/02:25 & Apr 21/23:20 & 2520 & $\mathrm{Halo} / 210127$ & $2393^{c}$ & Apr 21/01:51 & $\mathrm{X} 1 / 1 \mathrm{~F}$ & S14W84 & 9906 \\
\hline $44 \ldots \ldots \ldots . . .$. & 2002 & May 22/17:55 & May 23/10:55 & 820 & Halo/22 0326 & $1557^{\mathrm{c}}$ & May 22/03:54 & C5/DSF & $\mathrm{S} 30 \mathrm{~W} 34^{\mathrm{c}}$ & $\cdots$ \\
\hline $45 \ldots \ldots \ldots \ldots$ & 2002 & Jul 07/18:30 & Jul 07/19:55 & 22 & W/07 1106 & 1329 & Jul 07/11:43 & M1 & S W110 & 10017 \\
\hline $46 \ldots \ldots \ldots . .$. & 2002 & Jul 16/17:50 & Jul 17/16:00 & 234 & Halo/15 2030 & $1151^{\mathrm{c}}$ & Jul 15/20:08 & $\mathrm{X} 3 / 3 \mathrm{~B}$ & N19W01 & 10030 \\
\hline $47 \ldots \ldots \ldots$. & 2002 & Jul 22/06:55 & Jul 23/10:25 & 28 & Halo/20 2206 & 1941 & Jul 20/21:30 & $\mathrm{X} 3$ & SE90 & 10039 \\
\hline $48 \ldots \ldots \ldots \ldots$ & 2002 & Aug 14/09:00 & Aug 14/16:20 & 26 & $\mathrm{NW} / 140230$ & 1309 & Aug 14/02:12 & $\mathrm{M} 2 / 1 \mathrm{~N}$ & N09W54 & 10061 \\
\hline $49 \ldots \ldots \ldots$ & 2002 & Aug 24/01:40 & Aug 24/08:35 & 317 & $\mathrm{~W} / 240127$ & $1913^{\mathrm{c}}$ & Aug $24 / 01: 12$ & $\mathrm{X} 3 / 1 \mathrm{~F}$ & $\mathrm{~S} 02 \mathrm{~W} 81^{\mathrm{c}}$ & 10069 \\
\hline $50 \ldots \ldots \ldots \ldots$ & 2002 & Sep 07/04:40 & Sep 07/16:50 & 208 & Halo/05 1654 & 1748 & Sep 05/17:06 & C5/DSF & N09E28 & 10102 \\
\hline $51 \ldots \ldots \ldots . .$. & 2002 & Nov 09/19:20 & Nov 10/05:40 & 404 & SW/09 1331 & 1838 & Nov $09 / 13: 23$ & $\mathrm{M} 4 / 2 \mathrm{~B}$ & S12W29 & 10180 \\
\hline $52 \ldots \ldots \ldots$ & 2003 & May 28/23:35 & May 29/15:30 & 121 & Halo/28 0050 & 1366 & May 28/00:27 & $\mathrm{X} 3 / 2 \mathrm{~B}$ & S07W17 & 10365 \\
\hline $53 \ldots \ldots \ldots$. & 2003 & May $31 / 04: 40$ & May $31 / 06: 45$ & 27 & $\mathrm{~W} / 310230$ & 1835 & May 31/02:24 & $\mathrm{M} 9 / 2 \mathrm{~B}$ & S07W65 & 10365 \\
\hline $54 \ldots \ldots \ldots \ldots$ & 2003 & Jun 18/20:50 & June 19/04:50 & 24 & Halo/17 2318 & 1813 & Jun 17/22:55 & M6 & S08E61 & 10386 \\
\hline $55 \ldots \ldots \ldots$ & 2003 & Oct $26 / 18: 25$ & Oct $26 / 22: 35$ & 466 & Halo/26 1754 & 1537 & Oct $26 / 18: 19$ & $\mathrm{X} 1 / 1 \mathrm{~N}$ & N02W38 & 10484 \\
\hline
\end{tabular}


TABLE 1 - Continued

\begin{tabular}{|c|c|c|c|c|c|c|c|c|c|c|}
\hline \multicolumn{5}{|c|}{ NOAA GOES Particle Event } & \multicolumn{6}{|c|}{ Associated CME, Flare, and Active Region } \\
\hline SEP & Year & $\begin{array}{c}\text { Start Time } \\
\text { (month day/UT) }\end{array}$ & $\begin{array}{c}\text { Peak Time } \\
\text { (month day/UT) }\end{array}$ & $\begin{array}{l}\text { Peak Proton Flux } \\
\text { (pfu at }>10 \mathrm{MeV} \text { ) }\end{array}$ & $\begin{array}{l}\text { CME Location/ } \\
\text { Day-Time }\end{array}$ & $\begin{array}{l}\text { CME Speed } \\
\quad\left(\mathrm{km} \mathrm{s}^{-1}\right)\end{array}$ & $\begin{array}{c}\text { Maximum } \\
\text { (month day/UT) }\end{array}$ & Importance & Location & NOAA AR \\
\hline $56 \ldots$ & 2003 & Oct 28/12:15 & Oct 29/06:15 & 29500 & Halo/28 1130 & 2459 & Oct $28 / 11: 10$ & $\mathrm{X} 17 / 4 \mathrm{~B}$ & S16E08 & 10486 \\
\hline $57 \ldots \ldots \ldots \ldots . . . .$. & 2003 & Nov $04 / 22: 25$ & Nov 05/06:00 & 353 & Halo/04 1954 & 2657 & Nov 04/19:29 & $\mathrm{X} 28 / 3 \mathrm{~B}$ & S19W83 & 10486 \\
\hline $58 \ldots \ldots \ldots \ldots . . .$. & 2003 & Dec 02/15:05 & Dec 02/17:30 & 86 & Halo/02 1026 & 1393 & Dec 02/09:48 & $\mathrm{C} 7$ & $\mathrm{~S} 13 \mathrm{~W} 65^{\mathrm{b}}$ & 10508 \\
\hline $59 \ldots \ldots \ldots \ldots . . .$. & 2004 & Apr 11/11:35 & Apr 11/18:45 & 35 & $\mathrm{SW} / 110430$ & 1645 & Apr 11/04:19 & $\mathrm{C} 9 / 1 \mathrm{~F}$ & S14W47 & 10588 \\
\hline $62 \ldots \ldots \ldots \ldots \ldots$ & 2004 & Sep19/19:25 & Sep 20/01:00 & 57 & W/19 2224 & $\ldots$ & Sep 19/17:12 & M1 & N03W58 & 10672 \\
\hline $63 \ldots \ldots \ldots \ldots . .$. & 2004 & Nov $01 / 06: 55$ & Nov 01/08:05 & 63 & W/01 0606 & 790 & $\ldots$ & $\ldots$ & $\mathrm{W} 120^{\mathrm{b}}$ & 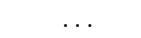 \\
\hline $64^{\mathrm{e}} \ldots \ldots \ldots \ldots$ & 2005 & Jan 20/06:30 & Jan 20/08:10 & 1860 & Halo/20 0654 & 3675 & Jan 20/07:01 & $\mathrm{X} 7$ & N14W61 & 10720 \\
\hline
\end{tabular}

NotEs.-(1) The following 15 NOAA SPEs were excluded because the ULEIS intensities were dominated by local ESP events or the event occurred during complex time periods: for 1997, November 6/13:05; for 1998, May 2/1420; for 2000, February 18/11:30, July 28/10:50, and August 11/16:50; for 2001, March 29/ 16:35, November 19/12:30, and December 29/05:10; for 2002, March 18/13:00, March 20/15:10, April 17/15:30, and July 19/10:50; for 2003, November 2/11:05 and November 21/23:55; and for 2004, November 7/19:10. (2) The following five events were excluded because ULEIS did not measure an appreciable increase in the O intensity: for 2001, September 15/14:35 and October 22/19:10; and for 2002, January 15/14:35, March 17/08:20, and August 22/04:40. (3) ULEIS was switched off during the Leonid meteor shower during the event on 1998 November 14/08:10.

${ }^{a}$ CME speeds from Gopalswamy et al. (2005a, 2005b) and http://cdaw.gsfc.nasa.gov/CME_list/UNIVERSAL/text_ver.

${ }^{\mathrm{b}}$ Flare locations from Cane et al. (2003, 2006).

c CME speeds and flare locations from Kahler (2005).

${ }^{\mathrm{d}}$ Flare/CME identifications from http://cdaw.gsfc.nasa.gov/LWS/data/text/AR_flare_radio_info.txt.

${ }^{\mathrm{e}}$ Last in a sequence of three SPEs that occurred between 2005 January 16-January 20.

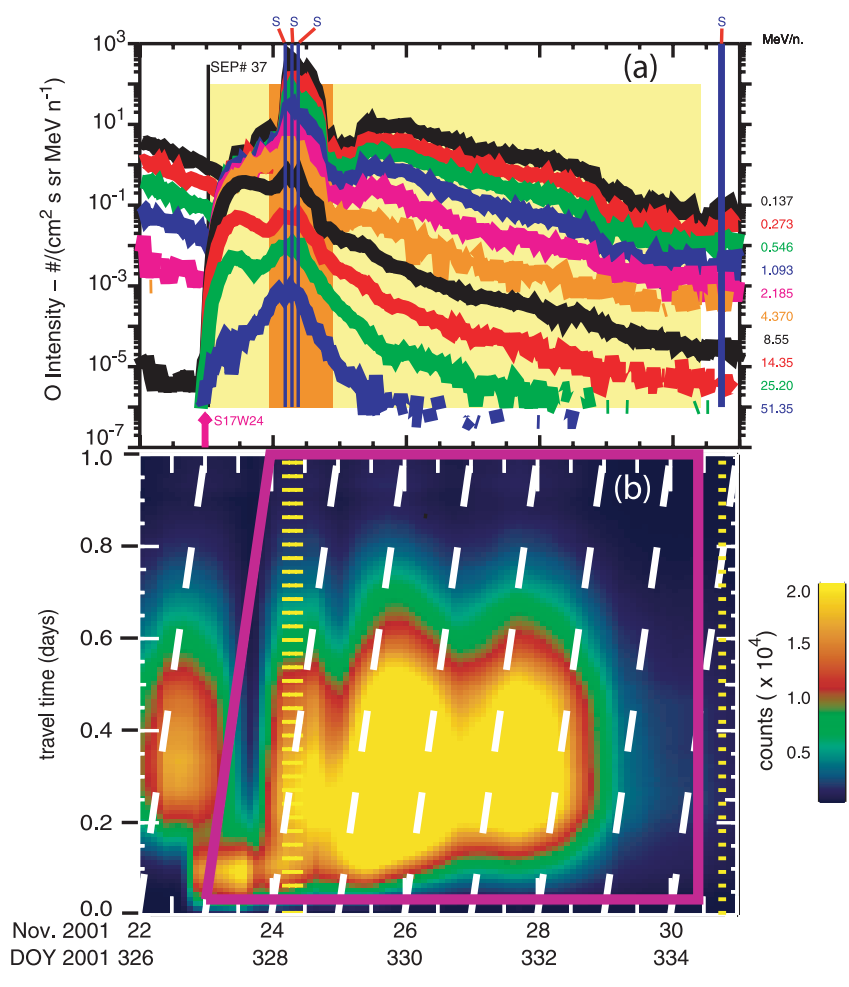

FIG. 1.-(a) Hourly averaged $\mathrm{O}$ intensities between $\sim 0.13$ and $55 \mathrm{MeV}$ nucleon $^{-1}$ measured by ULEIS and SIS for the period 2001 November 22-December 1, event 37 in Table 1: purple arrow, time of associated flare in AR 9704 at S15W34; shaded yellow interval, sampling interval for event 37 based on onset of $\sim 1 \mathrm{MeV}$ nucleon $^{-1}$ ions; orange shaded region, excluded time interval covering the ESP event; vertical blue line marked $S$, IP shock arrival times at $1 \mathrm{AU}$ at $A C E$. (b) Travel time (in days) vs. time for all ions detected by ULEIS: white diagonal lines, travel time for ions with $1.2 \mathrm{AU}$ path length along the Archimedian spiral; purple trapezoid, energy-dependent sampling interval for event 37; and vertical dashed lines, shock arrival times at 1 AU. mass histogram for nuclei with energies between 0.5 and 2.0 MeV nucleon ${ }^{-1}$. The background for ${ }^{4} \mathrm{He}$ is negligible, so the number of ${ }^{4} \mathrm{He}$ counts is obtained simply by summing over the peak. For ${ }^{3} \mathrm{He}$, background under the peak is estimated by linearly interpolating the number of counts on both sides of the ${ }^{3} \mathrm{He}$ peak (from $2.4-2.56$ and 3.2-3.28 amu). We then obtain the net number of ${ }^{3} \mathrm{He}$ counts by subtracting the interpolated background from the total number of counts in the mass range 2.6-3.16 amu. Note that the ${ }^{3} \mathrm{He}$ mass range is not symmetric

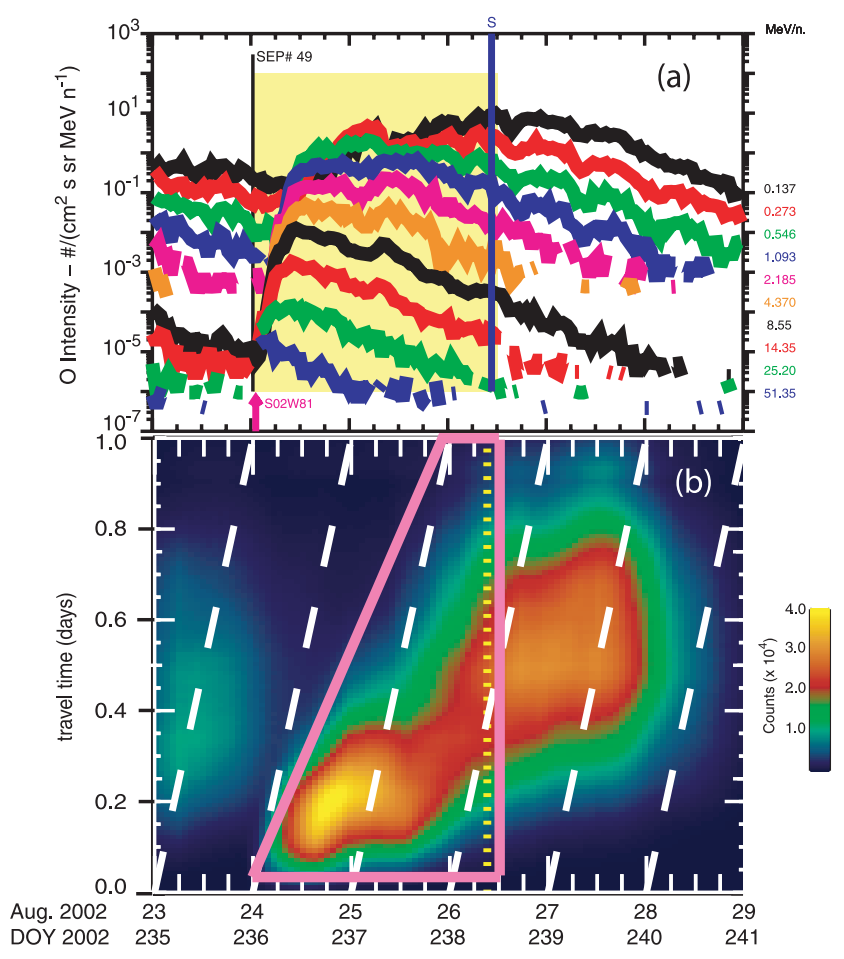

Fig. 2.- Same as Fig. 1, but for event 49 in Table 2 between August 23 and 29. 
TABLE 2

Sampling Intervals and Elemental Abundances for 64 Large Solar Particle Events

\begin{tabular}{|c|c|c|c|c|c|c|c|c|c|c|c|c|}
\hline \multirow[b]{2}{*}{ SEP } & \multirow[b]{2}{*}{ YEAR } & \multirow{2}{*}{$\begin{array}{l}\text { ULEIS SAMPLING INTERVALS } \\
\quad(\text { month day, UT })^{\mathrm{a}}\end{array}$} & \multirow{2}{*}{$\begin{array}{l}0.5-2 \mathrm{MeV} \text { nucleon }{ }^{-1} \\
{ }^{3} \mathrm{He} /{ }^{4} \mathrm{He}\left(\times 10^{3}\right)\end{array}$} & \multicolumn{9}{|c|}{$0.32-0.45 \mathrm{MeV}$ nucleon $^{-1}$} \\
\hline & & & & ${ }^{4} \mathrm{He} / \mathrm{O}$ & $\mathrm{C} / \mathrm{O}$ & $\mathrm{N} / \mathrm{O}$ & $\mathrm{Ne} / \mathrm{O}$ & $\mathrm{Mg} / \mathrm{O}$ & $\mathrm{Si} / \mathrm{O}$ & $\mathrm{S} / \mathrm{O}$ & $\mathrm{Ca} / \mathrm{O}$ & $\mathrm{Fe} / \mathrm{O}$ \\
\hline & 1997 & Nov 4, 12:05-Nov 6, 11:55 & $65 \pm 0.81$ & $56.6 \pm 39.1$ & $0.408 \pm 0.026$ & $0.123 \pm 0.012$ & $0.177 \pm 0.018$ & $0.237 \pm 0.018$ & $0.283 \pm 0.022$ & $0.090 \pm 0.011$ & $0.045 \pm 0.008$ & $0.862 \pm 0.041$ \\
\hline $2^{\mathrm{b}, \mathrm{c}} \ldots$ & 1998 & $\begin{array}{l}\text { Apr 20, 15:40-Apr 21, 00:00 } \\
\text { Apr 23, 19:10-Apr 26, 00:55 }\end{array}$ & $<1.21$ & $142.3 \pm 98.3$ & $0.363 \pm 0.023$ & $0.126 \pm 0.012$ & $0.169 \pm 0.014$ & $0.291 \pm 0.021$ & $0.217 \pm 0.018$ & $0.042 \pm 0.006$ & $0.012 \pm 0.004$ & $0.093 \pm 0.009$ \\
\hline ....... & 1998 & May 6, 11:55-May 7, 09:10 & $5.32 \pm 0.78$ & $131.2 \pm 90.6$ & $0.232 \pm 0.014$ & $0.099 \pm 0.008$ & $0.215 \pm 0.014$ & $0.233 \pm 0.014$ & $0.272 \pm 0.016$ & $0.085 \pm 0.008$ & $0.051 \pm 0.007$ & $0.830 \pm 0.034$ \\
\hline $4^{\mathrm{b}, \mathrm{c}} \ldots \ldots \ldots \ldots \ldots \ldots \ldots \ldots \ldots \ldots \ldots$ & 1998 & $\begin{array}{l}\text { Aug 25, 02:10-Aug 25, 22:40 } \\
\text { Aug 27, 04:05-Aug 28, 20:00 }\end{array}$ & $<0.39$ & $32.9 \pm 22.7$ & $0.369 \pm 0.013$ & $0.114 \pm 0.006$ & $0.172 \pm 0.008$ & $0.250 \pm 0.011$ & $0.222 \pm 0.009$ & $0.053 \pm 0.004$ & $0.015 \pm 0.002$ & $0.238 \pm 0.009$ \\
\hline $5^{\mathrm{b}, \mathrm{c}}$ & 1998 & $\begin{array}{l}\text { Sep 23, 04:55-Sep 24, 15:00 } \\
\text { Sep 25, 08:20-Sep 25, 22:45 }\end{array}$ & $5.84 \pm 0.90$ & $78.6 \pm 54.2$ & $0.523 \pm 0.023$ & $0.130 \pm 0.009$ & $0.145 \pm 0.010$ & $0.212 \pm 0.013$ & $0.149 \pm 0.010$ & $0.032 \pm 0.004$ & $0.005 \pm 0.002$ & $0.091 \pm 0.007$ \\
\hline $6^{\mathrm{c}} \ldots \ldots \ldots \ldots \ldots \ldots \ldots \ldots \ldots \ldots$ & 1998 & $\begin{array}{l}\text { Sep } 30,18: 35-\text { Oct } 2,04: 10 \\
\text { Oct } 2,11: 00-\text { Oct } 4,23: 00\end{array}$ & $<0.41$ & $56.8 \pm 39.2$ & $0.528 \pm 0.021$ & $0.128 \pm 0.008$ & $0.164 \pm 0.009$ & $0.203 \pm 0.010$ & $0.180 \pm 0.010$ & $0.040 \pm 0.004$ & $0.010 \pm 0.002$ & $0.195 \pm 0.006$ \\
\hline $7 .$. & 1998 & Nov $6,03: 00-\operatorname{Nov} 7,15: 30$ & $<0.52$ & $33.8 \pm 23.3$ & $0.320 \pm 0.010$ & $0.122 \pm 0.006$ & $0.151 \pm 0.006$ & $0.224 \pm 0.008$ & $0.143 \pm 0.005$ & $0.032 \pm 0.002$ & $0.006 \pm 0.001$ & $0.083 \pm 0.003$ \\
\hline & 1999 & Jan 21, 04:05-Jan 22, 14:40 & $<0.61$ & $63.6 \pm 43.9$ & $0.329 \pm 0.014$ & $0.115 \pm 0.007$ & $0.168 \pm 0.009$ & $0.194 \pm 0.010$ & $0.156 \pm 0.008$ & $0.036 \pm 0.003$ & $0.009 \pm 0.002$ & $0.097 \pm 0.005$ \\
\hline & 1999 & Apr $24,20: 05-$ Apr $26,15: 50$ & $<0.68$ & $43.6 \pm 13.5$ & $0.339 \pm 0.015$ & $0.120 \pm 0.008$ & $0.173 \pm 0.010$ & $0.211 \pm 0.011$ & $0.187 \pm 0.010$ & $0.048 \pm 0.005$ & $0.019 \pm 0.003$ & $0.186 \pm 0.010$ \\
\hline $10^{\mathrm{c}} \ldots \ldots \ldots \ldots \ldots \ldots \ldots \ldots \ldots \ldots$ & 1999 & $\begin{array}{l}\text { May 3, 19:45-May 5, 12:15 } \\
\text { May 6, 23:45-May 9, 12:25 }\end{array}$ & $<0.41$ & $104.0 \pm 32.3$ & $0.461 \pm 0.015$ & $0.131 \pm 0.006$ & $0.130 \pm 0.006$ & $0.214 \pm 0.008$ & $0.158 \pm 0.007$ & $0.034 \pm 0.003$ & $0.009 \pm 0.001$ & $0.110 \pm 0.004$ \\
\hline $11 \ldots \ldots \ldots \ldots \ldots \ldots \ldots$ & 1999 & Jun 1, 23:05-Jun 4, 08:40 & $25.50 \pm 1.71$ & $64.4 \pm 20.1$ & $0.291 \pm 0.018$ & $0.126 \pm 0.011$ & $0.189 \pm 0.014$ & $0.248 \pm 0.016$ & $0.268 \pm 0.018$ & $0.069 \pm 0.008$ & $0.034 \pm 0.006$ & $0.390 \pm 0.022$ \\
\hline $12 \ldots \ldots$ & 1999 & Jun 4, 11:25-Jun 8, 19:20 & $3.41 \pm 0.51$ & $35.8 \pm 11.1$ & $0.272 \pm 0.009$ & $0.106 \pm 0.005$ & $0.133 \pm 0.005$ & $0.271 \pm 0.009$ & $0.441 \pm 0.013$ & $0.139 \pm 0.005$ & $0.054 \pm 0.004$ & $1.063 \pm 0.027$ \\
\hline $13^{\mathrm{b}, \mathrm{c}} \ldots$ & 2000 & $\begin{array}{l}\text { Apr 4, 19:00-Apr 6, 07:30 } \\
\text { Apr 6, 23:40-Apr 7, 04:00 } \\
\text { Apr 7, 12:55-Apr 8, 11:05 }\end{array}$ & $0.94 \pm 0.46$ & $85.4 \pm 26.5$ & $0.329 \pm 0.013$ & $0.112 \pm 0.006$ & $0.163 \pm 0.008$ & $0.240 \pm 0.010$ & $0.250 \pm 0.011$ & $0.060 \pm 0.005$ & $0.025 \pm 0.003$ & $0.373 \pm 0.011$ \\
\hline $14^{\mathrm{b}, \mathrm{c}}$. & 2000 & $\begin{array}{l}\text { Jun } 6,20: 25-\text { Jun } 8,03: 10 \\
\text { Jun } 8,16: 20-\text { Jun } 10,01: 45\end{array}$ & $<0.55$ & $78.5 \pm 24.4$ & $0.406 \pm 0.014$ & $0.125 \pm 0.006$ & $0.140 \pm 0.006$ & $0.191 \pm 0.008$ & $0.157 \pm 0.007$ & $0.030 \pm 0.003$ & $0.008 \pm 0.001$ & $0.111 \pm 0.003$ \\
\hline $15 \ldots$. & 2000 & Jun $10,21: 05-$ Jun $11,21: 10$ & $<0.92$ & $64.4 \pm 20.1$ & $0.375 \pm 0.024$ & $0.131 \pm 0.013$ & $0.118 \pm 0.012$ & $0.307 \pm 0.021$ & $0.333 \pm 0.023$ & $0.077 \pm 0.009$ & $0.031 \pm 0.007$ & $0.552 \pm 0.026$ \\
\hline $16^{\mathrm{b}, \mathrm{c}}$. & 2000 & Jul 15, 22:05-Jul 19, 04:40 & $<0.38$ & $173.3 \pm 53.8$ & $0.381 \pm 0.014$ & $0.120 \pm 0.006$ & $0.190 \pm 0.009$ & $0.259 \pm 0.011$ & $0.219 \pm 0.010$ & $0.050 \pm 0.004$ & $0.020 \pm 0.003$ & $0.267 \pm 0.008$ \\
\hline ......... & 2000 & Jul 22, 16:35-Jul 23, 20:15 & $3.33 \pm 0.98$ & $93.7 \pm 29.2$ & $0.295 \pm 0.017$ & $0.116 \pm 0.010$ & $0.193 \pm 0.013$ & $0.277 \pm 0.017$ & $0.217 \pm 0.014$ & $0.033 \pm 0.005$ & $0.015 \pm 0.004$ & $0.155 \pm 0.009$ \\
\hline 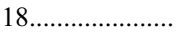 & 2000 & Sep 12, 17:20-Sep 16, 14:00 & $<0.45$ & $67.6 \pm 21.0$ & $0.305 \pm 0.009$ & $0.115 \pm 0.004$ & $0.127 \pm 0.005$ & $0.153 \pm 0.005$ & $0.186 \pm 0.006$ & $0.059 \pm 0.003$ & $0.017 \pm 0.002$ & $0.290 \pm 0.007$ \\
\hline $19 \ldots \ldots$. & 2000 & Oct $16,13: 30-\mathrm{O}$ & $96 \pm 1.1$ & 111 & $0.262 \pm$ & $0.097 \pm$ & $0.159 \pm$ & 0.1 & $8 \pm$ & $0.101=$ & 08 & $0.609=$ \\
\hline $20 \ldots \ldots \ldots \ldots \ldots \ldots$ & 2000 & Oct $25,16: 35-$ Oct $28,02: 30$ & $6.14 \pm 0.77$ & $37.7 \pm 11.7$ & $0.335 \pm 0.013$ & $0.116 \pm 0.006$ & $0.108 \pm 0.006$ & $0.206 \pm 0.009$ & $0.212 \pm 0.010$ & $0.046 \pm 0.004$ & $0.014 \pm 0.002$ & $0.215 \pm 0.007$ \\
\hline $21^{\mathrm{b}, \mathrm{c}} \ldots \ldots \ldots \ldots \ldots$ & 2000 & $\begin{array}{l}\text { Nov } 9,15: 35-\text { Nov } 10,02: 40 \\
\text { Nov } 10,09: 40-\text { Nov 10, 16:20 } \\
\text { Nov } 11,07: 40-\text { Nov } 14,22: 20\end{array}$ & $<14.58$ & $68.1 \pm 21.3$ & $0.572 \pm 0.034$ & $0.152 \pm 0.015$ & $0.150 \pm 0.014$ & $0.173 \pm 0.016$ & $0.144 \pm 0.014$ & $0.038 \pm 0.006$ & $0.013 \pm 0.004$ & $0.236 \pm 0.012$ \\
\hline $22^{\mathrm{b}, \mathrm{c}, \mathrm{d}}$. & 2000 & $\begin{array}{l}\text { Nov } 24,12: 15-\text { Nov } 26,02: 00 \\
\text { Nov 26, 17:20-Nov 26, 19:10 } \\
\text { Nov 27, 07:00-Nov 28, 00:40 } \\
\text { Nov 28, 09:40-Nov 28, 22:20 } \\
\text { Nov 29, 14:20-Nov 29, 21:00 }\end{array}$ & $2.96 \pm 0.50$ & $44.7 \pm 13.9$ & $0.303 \pm 0.014$ & $0.124 \pm 0.008$ & $0.172 \pm 0.010$ & $0.204 \pm 0.011$ & $0.233 \pm 0.013$ & $0.078 \pm 0.006$ & $0.019 \pm 0.003$ & $0.407 \pm 0.013$ \\
\hline & 2001 & Jan 29, 01:00-Feb 1, 04:45 & $<0.42$ & $102.5 \pm 31.8$ & $0.352 \pm$ & $0.121 \pm 0.005$ & $0.111 \pm 0.005$ & $0.147=$ & $0.191 \pm$ & $0.060 \pm$ & $0.017=$ & $0.274 \pm 0.007$ \\
\hline $24^{\mathrm{e}} \ldots \ldots \ldots \ldots \ldots \ldots$ & 2001 & $\begin{array}{l}\text { Apr 3, 01:55-Apr 4, 01:20 } \\
\text { Apr 4, 21:45-Apr 5, 11:00 }\end{array}$ & $4.20 \pm 0.76$ & $167.0 \pm 51.9$ & $0.367 \pm 0.019$ & $0.107 \pm 0.009$ & $0.197 \pm 0.013$ & $0.285 \pm 0.016$ & $0.247 \pm 0.015$ & $0.052 \pm 0.006$ & $0.022 \pm 0.004$ & $0.456 \pm 0.022$ \\
\hline $25^{\mathrm{b}, \mathrm{c}} \ldots$ & 2001 & Apr 10, 11:10-Apr 11, 08:10 & $<0.83$ & $89.8 \pm 28.0$ & $0.449 \pm 0.024$ & $0.148 \pm 0$ & $0.115 \pm 0.010$ & $0.227 \pm 0$ & $0.298 \pm 0.019$ & $0.088 \pm 0.009$ & $0.025 \pm$ & $0.572 \pm 0.022$ \\
\hline $26 \ldots \ldots \ldots \ldots \ldots \ldots \ldots$ & 2001 & Apr $15,18: 40-$ Apr 17, 17:50 & $<0.63$ & $123.4 \pm 38.4$ & $0.290 \pm 0.014$ & $0.107 \pm 0.008$ & $0.164 \pm 0.010$ & $0.286 \pm 0.014$ & $0.376 \pm 0.018$ & $0.113 \pm 0.008$ & $0.047 \pm 0.006$ & $1.325 \pm 0.047$ \\
\hline $27^{\mathrm{c}} \ldots \ldots \ldots \ldots \ldots \ldots \ldots$ & 2001 & $\begin{array}{l}\text { Apr 18, 06:05-Apr 21, 10:05 } \\
\text { Apr 21, 19:30-Apr 21, 21:50 }\end{array}$ & $<0.96$ & $66.9 \pm 20.8$ & $0.344 \pm 0.015$ & $0.118 \pm 0.008$ & $0.176 \pm 0.010$ & $0.271 \pm 0.013$ & $0.238 \pm 0.013$ & $0.044 \pm 0.005$ & $0.014 \pm 0.003$ & $0.206 \pm 0.011$ \\
\hline $28^{\mathrm{b}, \mathrm{c}} \ldots$ & 2001 & $\begin{array}{l}\text { Apr } 26,17: 45-\text { Apr } 27,15: 15 \\
\text { Apr } 29,03: 40-\text { Apr } 30,01: 45\end{array}$ & $2.50 \pm 0.85$ & $85.3 \pm 26.5$ & $0.372 \pm 0.018$ & $0.127 \pm 0.009$ & $0.148 \pm 0.010$ & $0.233 \pm 0.013$ & $0.135 \pm 0.010$ & $0.021 \pm 0.004$ & $0.005 \pm 0.001$ & $0.071 \pm 0.004$ \\
\hline
\end{tabular}


TABLE 2-Continued

\begin{tabular}{|c|c|c|c|c|c|c|c|c|c|c|c|c|}
\hline \multirow[b]{2}{*}{ SEP } & \multirow[b]{2}{*}{ YeAR } & \multirow{2}{*}{$\begin{array}{l}\text { ULEIS SAMPLING INTERVALS } \\
\quad(\text { month day, UT })^{\mathrm{a}}\end{array}$} & \multirow{2}{*}{$\begin{array}{l}0.5-2 \mathrm{MeV} \text { nucleon }{ }^{-1} \\
{ }^{3} \mathrm{He} /{ }^{4} \mathrm{He}\left(\times 10^{3}\right)\end{array}$} & \multicolumn{9}{|c|}{$0.32-0.45 \mathrm{MeV}$ nucleon $^{-1}$} \\
\hline & & & & ${ }^{4} \mathrm{He} / \mathrm{O}$ & $\mathrm{C} / \mathrm{O}$ & $\mathrm{N} / \mathrm{O}$ & $\mathrm{Ne} / \mathrm{O}$ & $\mathrm{Mg} / \mathrm{O}$ & $\mathrm{Si} / \mathrm{O}$ & $\mathrm{S} / \mathrm{O}$ & $\mathrm{Ca} / \mathrm{O}$ & $\mathrm{Fe} / \mathrm{O}$ \\
\hline & 2001 & May 7, 15:35-May 9, 09:50 & $4.29 \pm 0.77$ & $80.1 \pm 24.9$ & $0.285 \pm 0.016$ & $0.116 \pm 0.009$ & $0.162 \pm 0.011$ & $0.211 \pm 0.013$ & $0.270 \pm 0.016$ & $0.062 \pm 0.007$ & $0.033 \pm 0.005$ & $0.698 \pm 0.030$ \\
\hline & 2001 & Jun $15,21: 35-$ Jun $17,18: 30$ & 0.85 & $112.8 \pm$ & $0.274 \pm$ & $0.102 \pm 0.007$ & $0.144 \pm 0.009$ & $0.214 \pm 0.011$ & $0.234 \pm 0.012$ & $0.047 \pm 0.005$ & $0.019 \pm 0.003$ & $0.216 \pm 0.009$ \\
\hline $31^{\mathrm{c}}$. & 2001 & $\begin{array}{l}\text { Aug 9, 17:35-Aug 12, 06:50 } \\
\text { Aug 12, 22:00-Aug 14, 13:40 }\end{array}$ & $1.34 \pm 0.55$ & $94.1 \pm 29.2$ & $0.300 \pm 0.010$ & $0.115 \pm 0.005$ & $0.139 \pm 0.006$ & $0.173 \pm 0.007$ & $0.163 \pm 0.007$ & $0.036 \pm 0.003$ & $0.009 \pm 0.001$ & $0.108 \pm 0.003$ \\
\hline $32^{\mathrm{c}} \ldots \ldots \ldots \ldots \ldots \ldots$ & 2001 & $\begin{array}{l}\text { Aug 16, 04:20-Aug 17, 06:00 } \\
\text { Aug 17, 16:25-Aug 18, 04:25 }\end{array}$ & $2.33 \pm 0.91$ & $66.4 \pm 20.6$ & $0.340 \pm 0.015$ & $0.116 \pm 0.008$ & $0.152 \pm 0.009$ & $0.231 \pm 0.012$ & $0.222 \pm 0.012$ & $0.041 \pm 0.004$ & $0.013 \pm 0.002$ & $0.157 \pm 0.007$ \\
\hline $33^{\mathrm{b}, \mathrm{c}} \ldots$ & 2001 & $\begin{array}{l}\text { Sep } 24,15: 35-\text { Sep } 25,15: 00 \\
\text { Sep 27, 12:00-Sep 30, 22:30 }\end{array}$ & $<0.52$ & $69.0 \pm 21.4$ & $0.377 \pm 0.011$ & $0.117 \pm 0.005$ & $0.133 \pm 0.005$ & $0.237 \pm 0.008$ & $0.243 \pm 0.008$ & $0.055 \pm 0.003$ & $0.023 \pm 0.002$ & $0.297 \pm 0.008$ \\
\hline $34^{\mathrm{c}} \ldots \ldots \ldots \ldots \ldots \ldots \ldots \ldots$ & 2001 & $\begin{array}{l}\text { Oct } 1,11: 35 \text {-Oct } 2,05: 35 \\
\text { Oct } 3,23: 10 \text {-Oct } 6,00: 00\end{array}$ & $1.08 \pm 0.50$ & $46.4 \pm 14.4$ & $0.314 \pm 0.014$ & $0.124 \pm 0.008$ & $0.162 \pm 0.009$ & $0.237 \pm 0.012$ & $0.182 \pm 0.010$ & $0.026 \pm 0.003$ & $0.010 \pm 0.003$ & $0.097 \pm 0.004$ \\
\hline & 2001 & Oct $20,00: 10$-Oct $20,21: 20$ & $1.78 \pm$ & $50.8 \pm$ & $0.270 \pm 0.016$ & $0.108 \pm 0.009$ & $0.139 \pm 0.011$ & $0.246 \pm 0.015$ & $0.306 \pm 0.018$ & $0.071 \pm 0.008$ & $0.025 \pm 0.005$ & $0.438 \pm 0.021$ \\
\hline ( & 2001 & $\begin{array}{l}\text { Nov } 4,20: 35-\operatorname{Nov} 5,21: 35 \\
\text { Nov } 6,20: 10-\operatorname{Nov} 8,23: 00\end{array}$ & $<3.05$ & $37.6 \pm 11.7$ & $0.462 \pm 0.028$ & $0.130 \pm 0.013$ & $0.126 \pm 0.012$ & $0.221 \pm 0.017$ & $0.173 \pm 0.016$ & $0.045 \pm 0.008$ & $0.016 \pm 0.004$ & $0.176 \pm 0.016$ \\
\hline $37^{\mathrm{c}} \ldots \ldots \ldots \ldots \ldots \ldots$ & 2001 & $\begin{array}{l}\text { Nov } 23,03: 10-\text { Nov } 23,22: 30 \\
\text { Nov } 24,21: 15-\text { Nov } 30,10: 00\end{array}$ & $<0.87$ & $49.1 \pm 15.2$ & $0.360 \pm 0.015$ & $0.103 \pm 0.006$ & $0.152 \pm 0.008$ & $0.273 \pm 0.012$ & $0.301 \pm 0.014$ & $0.064 \pm 0.005$ & $0.028 \pm 0.004$ & $0.502=$ \\
\hline $38 \ldots$ & 2001 & $\operatorname{Dec} 26,08: 35-\operatorname{Dec} 28,18: 00$ & $<0.42$ & $39.7 \pm 12.3$ & $0.319 \pm 0.010$ & $0.112 \pm 0.005$ & $0.139 \pm 0.006$ & $0.207 \pm$ & $0.260 \pm$ & $0.079 \pm 0.004$ & $0.025=$ & $0.646=$ \\
\hline $39 \ldots \ldots \ldots \ldots \ldots \ldots \ldots \ldots$ & 2001 & Dec 30, 22:45-Jan 7, 23:30 & $1.05 \pm 0.30$ & $55.0 \pm 17.2$ & $0.362 \pm 0.024$ & $0.139 \pm 0.013$ & $0.145 \pm 0.014$ & $0.205 \pm 0.016$ & $0.188 \pm 0.016$ & $0.053 \pm 0.008$ & $0.020 \pm 0.005$ & $0.312 \pm 0.020$ \\
\hline $40 \ldots \ldots \ldots \ldots \ldots \ldots \ldots \ldots$ & 2002 & Jan $10,19: 20-\operatorname{Jan} 14,00: 20$ & $<0.44$ & $94.9 \pm 29.5$ & $0.527 \pm 0.016$ & $0.142 \pm 0.006$ & $0.153 \pm 0.006$ & $0.164 \pm 0.007$ & $0.116 \pm 0.006$ & $0.028 \pm 0.002$ & $0.007 \pm 0.001$ & $0.080 \pm 0.003$ \\
\hline 41.......................... & 2002 & Feb 20, 09:35-Feb 22, 05:50 & $23.01 \pm 1.42$ & $100.4 \pm 31.2$ & $0.340 \pm 0.017$ & $0.102 \pm 0.008$ & $0.195 \pm 0.012$ & $0.326 \pm 0.017$ & $0.404 \pm 0.021$ & $0.118 \pm 0.010$ & $0.066 \pm 0.008$ & $1.489 \pm 0.057$ \\
\hline $42^{\mathrm{c}} \ldots \ldots \ldots \ldots \ldots \ldots$ & 2002 & $\begin{array}{l}\text { Mar 22, 16:20-Mar 23, 08:45 } \\
\text { Mar 23, 23:25-Mar 26, 11:10 }\end{array}$ & $1.66 \pm 0.46$ & $40.4 \pm 12.5$ & $0.370 \pm 0.013$ & $0.130 \pm 0.006$ & $0.129 \pm 0.006$ & $0.252 \pm 0.010$ & $0.259 \pm 0.011$ & $0.048 \pm 0.004$ & $0.022 \pm 0.003$ & $0.268 \pm 0.008$ \\
\hline $43^{\mathrm{c}} \ldots \ldots \ldots \ldots \ldots \ldots$ & 2002 & $\begin{array}{l}\text { Apr 21, 05:35-Apr 22, 21:30 } \\
\text { Apr 23, 19:25-Apr 24, 18:00 }\end{array}$ & 191 & $28.0 \pm 8.7$ & $0.319 \pm 0.022$ & $0.116 \pm 0.012$ & $0.099 \pm 0.011$ & $0.298 \pm 0.022$ & $0.329 \pm 0.024$ & $0.080 \pm 0.011$ & $0.037 \pm 0.007$ & $0.752 \pm 0.043$ \\
\hline $44^{\mathrm{c}}$ & 2002 & $\begin{array}{l}\text { May } 22,08: 35-\text { May } 23,05: 20 \\
\text { May } 23,18: 15-\text { May } 24,23: 35\end{array}$ & $<1.76$ & $56.1 \pm 17.4$ & $0.392 \pm 0.015$ & $0.122 \pm 0.007$ & $0.140 \pm 0.007$ & $0.188 \pm 0.009$ & $0.159 \pm 0.009$ & $0.041 \pm 0.004$ & $0.009 \pm 0.001$ & $0.128 \pm 0.006$ \\
\hline 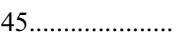 & 2002 & Jul 7, 15:30-Jul 10, 12:15 & $1.65=$ & $41.5 \pm$ & $0.262 \pm$ & $0.112 \pm$ & $0.161 \pm 0.007$ & $0.147 \pm$ & $0.145 \pm$ & $0.026 \pm$ & 0.005 & $0.028 \pm 0.002$ \\
\hline $46^{\mathrm{c}} \ldots$ & 2002 & $\begin{array}{l}\text { Jul 16, 07:00-Jul 17, 13:05 } \\
\text { Jul 17, 21:05-Jul 18, 18:35 }\end{array}$ & $<0.57$ & $83.2 \pm 25.8$ & $0.381 \pm 0.016$ & $0.115 \pm 0.007$ & $0.125 \pm 0.008$ & $0.196 \pm 0.010$ & $0.205 \pm 0.011$ & $0.043 \pm 0.005$ & $0.016 \pm 0.003$ & $0.188 \pm 0.009$ \\
\hline $47^{\mathrm{c}} \ldots \ldots \ldots \ldots \ldots \ldots$ & 2002 & $\begin{array}{l}\text { Jul 22, 05:05-Jul 25, 00:55 } \\
\text { Jul 26, 00:20-Jul 29, 03:20 }\end{array}$ & $0.79 \pm 0.33$ & $81.9 \pm 25.4$ & $0.368 \pm 0.010$ & $0.120 \pm 0.004$ & $0.128 \pm 0.005$ & $0.213 \pm 0.007$ & $0.178 \pm 0.006$ & $0.033 \pm 0.002$ & $0.008 \pm 0.001$ & $0.127 \pm 0.004$ \\
\hline
\end{tabular}


TABLE 2-Continued

\begin{tabular}{|c|c|c|c|c|c|c|c|c|c|c|c|c|}
\hline \multirow[b]{2}{*}{ SEP } & \multirow[b]{2}{*}{ YEAR } & \multirow{2}{*}{$\begin{array}{l}\text { ULEIS SAMPLING INTERVALS } \\
\quad(\text { month day, UT })^{\mathrm{a}}\end{array}$} & \multirow{2}{*}{$\begin{array}{l}0.5-2 \mathrm{MeV} \text { nucleon }{ }^{-1} \\
{ }^{3} \mathrm{He} /{ }^{4} \mathrm{He}\left(\times 10^{3}\right)\end{array}$} & \multicolumn{9}{|c|}{$0.32-0.45 \mathrm{MeV}$ nucleon $^{-1}$} \\
\hline & & & & ${ }^{4} \mathrm{He} / \mathrm{O}$ & $\mathrm{C} / \mathrm{O}$ & $\mathrm{N} / \mathrm{O}$ & $\mathrm{Ne} / \mathrm{O}$ & $\mathrm{Mg} / \mathrm{O}$ & $\mathrm{Si} / \mathrm{O}$ & $\mathrm{S} / \mathrm{O}$ & $\mathrm{Ca} / \mathrm{O}$ & $\mathrm{Fe} / \mathrm{O}$ \\
\hline $48 \ldots \ldots \ldots \ldots \ldots \ldots \ldots$ & 2002 & Aug 14, 05:00-Aug 15, 19:50 & $11.96 \pm 0.99$ & $51.2 \pm 15.9$ & $0.392 \pm 0.018$ & $0.124 \pm 0.008$ & $0.141 \pm 0.009$ & $0.281 \pm 0.014$ & $0.275 \pm 0.015$ & $0.061 \pm 0.006$ & $0.018 \pm 0.004$ & $0.253 \pm 0.009$ \\
\hline $49 \ldots \ldots \ldots \ldots \ldots \ldots$ & 2002 & Aug 24, 05:25-Aug 26, 12:00 & $1.82 \pm 0.46$ & $52.9 \pm 16.4$ & $0.336 \pm 0.011$ & $0.114 \pm 0.005$ & $0.138 \pm 0.006$ & $0.251 \pm 0.009$ & $0.285 \pm 0.010$ & $0.077 \pm 0.004$ & $0.027 \pm 0.003$ & $0.536 \pm 0.014$ \\
\hline $50^{\mathrm{c}} \ldots \ldots \ldots \ldots \ldots \ldots \ldots$ & 2002 & $\begin{array}{c}\text { Sep 6, 0105-Sep 7, 13:20 } \\
\text { Sep 8, 03:00-Sep 12, 23:25 }\end{array}$ & $1.89 \pm 0.49$ & $111.9 \pm 34.8$ & $0.428 \pm 0.020$ & $0.128 \pm 0.009$ & $0.166 \pm 0.011$ & $0.248 \pm 0.013$ & $0.219 \pm 0.013$ & $0.030 \pm 0.004$ & $0.009 \pm 0.002$ & $0.143 \pm 0.010$ \\
\hline $51 \ldots \ldots \ldots \ldots \ldots \ldots \ldots$ & 2002 & Nov 9, 19:40-Nov 11, 07:00 & $<1.22$ & $92.8 \pm 28.9$ & $0.389 \pm 0.025$ & $0.120 \pm 0.012$ & $0.172 \pm 0.014$ & $0.319 \pm 0.021$ & $0.339 \pm 0.023$ & $0.103 \pm 0.011$ & $0.026 \pm 0.005$ & $0.754 \pm 0.031$ \\
\hline $52^{\mathrm{c}} \ldots \ldots \ldots \ldots \ldots \ldots \ldots$ & 2003 & $\begin{array}{l}\text { May 28, 05:35-May 29, 10:25 } \\
\text { May 30, 04:00-May 30, 11:20 } \\
\text { May 31, 02:20-May 31, 07:20 }\end{array}$ & $<0.40$ & $101.4 \pm 31.5$ & $0.463 \pm 0.016$ & $0.129 \pm 0.007$ & $0.177 \pm 0.008$ & $0.197 \pm 0.009$ & $0.221 \pm 0.010$ & $0.051 \pm 0.004$ & $0.019 \pm 0.003$ & $0.328 \pm 0.011$ \\
\hline $53 \ldots \ldots \ldots \ldots \ldots \ldots \ldots$ & 2003 & May 31, 08:10-Jun 2, 02:05 & $<0.78$ & $52.2 \pm 16.2$ & $0.356 \pm 0.015$ & $0.122 \pm 0.007$ & $0.139 \pm 0.008$ & $0.228 \pm 0.011$ & $0.206 \pm 0.011$ & $0.059 \pm 0.005$ & $0.017 \pm 0.003$ & $0.304 \pm 0.012$ \\
\hline $54 \ldots \ldots \ldots \ldots \ldots \ldots$ & 2003 & Jun $18,05: 55-J u n ~ 23,23: 20$ & $<0.28$ & $106.4 \pm 33.0$ & $0.511 \pm 0.013$ & $0.148 \pm 0.005$ & $0.145 \pm 0.005$ & $0.215 \pm 0.006$ & $0.161 \pm 0.005$ & $0.030 \pm 0.002$ & $0.010 \pm 0.001$ & $0.126 \pm 0.004$ \\
\hline ……....... & 2003 & Oct $26,21: 20-$ Oct $27,20: 30$ & $1.67 \pm 0.80$ & $84.0 \pm 26.2$ & $0.303 \pm 0.022$ & $0.119 \pm 0.012$ & $0.200 \pm 0.017$ & $0.278 \pm 0.021$ & $0.292 \pm 0.023$ & $0.121 \pm 0.014$ & $0.058 \pm 0.010$ & $1.701 \pm 0.075$ \\
\hline $56^{\mathrm{b}, \mathrm{c}} \ldots \ldots \ldots \ldots \ldots \ldots$ & 2003 & Oct 29 14:30 - Oct 30, 00:00 & $<2.39$ & $72.6 \pm 22.9$ & $0.342 \pm 0.038$ & $0.113 \pm 0.019$ & $0.160 \pm 0.023$ & $0.171 \pm 0.025$ & $0.196 \pm 0.028$ & $0.042 \pm 0.013$ & $0.019 \pm 0.009$ & $0.200 \pm 0.029$ \\
\hline $57 \ldots \ldots \ldots \ldots \ldots \ldots \ldots$ & 2003 & Nov $5,01: 10-\operatorname{Nov} 9,15: 25$ & $<0.42$ & $40.6 \pm 12.6$ & $0.298 \pm 0.009$ & $0.107 \pm 0.004$ & $0.097 \pm 0.004$ & $0.250 \pm 0.008$ & $0.422 \pm 0.012$ & $0.114 \pm 0.005$ & $0.043 \pm 0.003$ & $1.217 \pm 0.030$ \\
\hline .............. & 2003 & Dec 2, 14:55-Dec 6, 03:00 & $0.85 \pm 0.45$ & $53.0 \pm 16.4$ & $0.248 \pm 0.008$ & $0.104 \pm 0.005$ & $0.134 \pm 0.006$ & $0.216 \pm 0.008$ & $0.313 \pm 0.010$ & $0.094 \pm 0.005$ & $0.034 \pm 0.003$ & $0.887 \pm 0.022$ \\
\hline $59^{\mathrm{c}} \ldots \ldots \ldots \ldots \ldots \ldots \ldots \ldots \ldots \ldots$ & 2004 & $\begin{array}{l}\text { Apr 11, 10:50-Apr 12, 12:00 } \\
\text { Apr 12, 20:50-Apr 13, 10:10 }\end{array}$ & $<0.63$ & $34.1 \pm 10.6$ & $0.362 \pm 0.015$ & $0.122 \pm 0.007$ & $0.123 \pm 0.007$ & $0.196 \pm 0.010$ & $0.236 \pm 0.012$ & $0.052 \pm 0.005$ & $0.015 \pm 0.002$ & $0.316 \pm 0.010$ \\
\hline $60^{\mathrm{c}} \ldots \ldots \ldots \ldots \ldots \ldots$ & 2004 & $\begin{array}{l}\text { Jul 25, 19:25-Jul 26, 14:30 } \\
\text { Jul 27, 05:20-Jul 28, 20:10 }\end{array}$ & $<0.45$ & $110.8 \pm 34.4$ & $0.420 \pm 0.014$ & $0.127 \pm 0.006$ & $0.157 \pm 0.007$ & $0.284 \pm 0.010$ & $0.255 \pm 0.010$ & $0.051 \pm 0.004$ & $0.025 \pm 0.002$ & $0.356 \pm 0.011$ \\
\hline 61........................ & 2004 & Sep 13, 12:00-Sep 17, 12:00 & $<0.36$ & $74.2 \pm 23.0$ & $0.421 \pm 0.014$ & $0.133 \pm 0.006$ & $0.122 \pm 0.006$ & $0.190 \pm 0.008$ & $0.161 \pm 0.007$ & $0.032 \pm 0.003$ & $0.008 \pm 0.001$ & $0.140 \pm 0.005$ \\
\hline $62 \ldots \ldots \ldots \ldots \ldots \ldots \ldots$ & 2004 & Sep 20, 00:15-Sep 24, 13:15 & $<0.61$ & $79.4 \pm 24.7$ & $0.335 \pm 0.018$ & $0.103 \pm 0.008$ & $0.109 \pm 0.008$ & $0.167 \pm 0.011$ & $0.179 \pm 0.012$ & $0.047 \pm 0.005$ & $0.009 \pm 0.002$ & $0.232 \pm 0.009$ \\
\hline 63....................... & 2004 & Nov $1,10: 30-\operatorname{Nov} 3,18: 00$ & $60.64 \pm 4.94$ & $94.0 \pm 29.5$ & $0.317 \pm 0.030$ & $0.111 \pm 0.016$ & $0.270 \pm 0.027$ & $0.353 \pm 0.032$ & $0.424 \pm 0.038$ & $0.114 \pm 0.017$ & $0.080 \pm 0.014$ & $1.051 \pm 0.072$ \\
\hline $64^{\mathrm{c}} \ldots \ldots \ldots \ldots \ldots \ldots \ldots$ & 2005 & $\begin{array}{l}\text { Jan } 20,11: 45-\operatorname{Jan} 21,09: 00 \\
\text { Jan 21, 22:00-Jan 24, 20:45 }\end{array}$ & $<0.77$ & $64.6 \pm 20.1$ & $0.381 \pm 0.020$ & $0.104 \pm 0.009$ & $0.147 \pm 0.011$ & $0.152 \pm 0.011$ & $0.150 \pm 0.013$ & $0.044 \pm 0.007$ & $0.018 \pm 0.005$ & $0.261 \pm 0.021$ \\
\hline
\end{tabular}

a Sampling times based on onset of $\sim 1 \mathrm{MeV}$ nucleon $^{-1}$ ion intensity.

${ }^{b}$ Excluded portions when ULEIS suffered loss in efficiency or from saturation effects.

${ }^{c}$ Excluded time intervals around the passage of IP shocks at $A C E$.

d Sampling interval may contain ions from multiple injections (see Kahler 2005). 

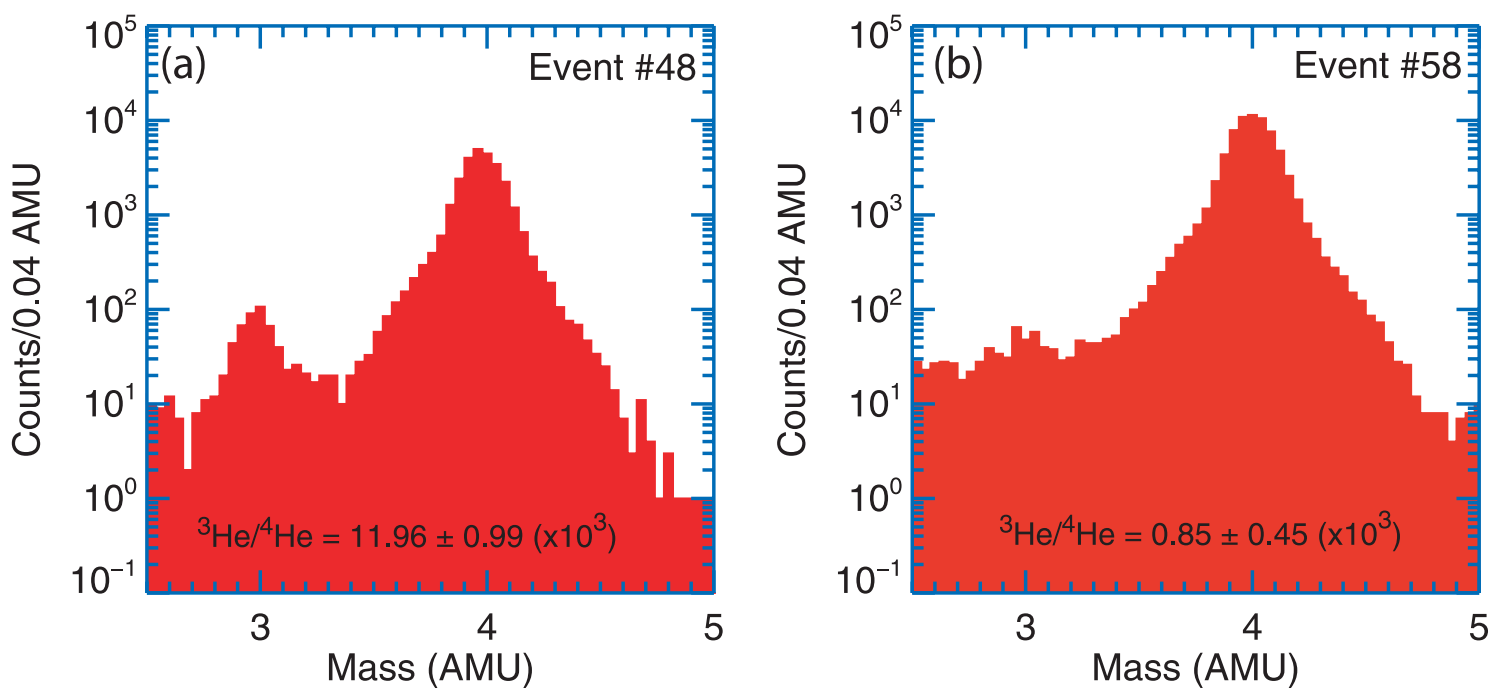

Fig. 3. - The 0.5-2.0 MeV nucleon ${ }^{-1}$ helium mass histograms for events 48 and 58 . The ${ }^{3} \mathrm{He}$ peak is finite and well resolved from the ${ }^{4} \mathrm{He}$ peak for event 48 . Although the background in event 58 is substantially higher, the presence of ${ }^{3} \mathrm{He}$ is still clear. The ${ }^{3} \mathrm{He} /{ }^{4} \mathrm{He}$ abundance ratios and their uncertainties are also shown.

about mass 3.0 due to the presence of the ${ }^{4} \mathrm{He}$ peak. The statistical uncertainty on the net number of ${ }^{3} \mathrm{He}$ counts was conservatively calculated as the square root of the total number of counts (including background) in the ${ }^{3} \mathrm{He}$ mass range.

In determining whether there is a finite ${ }^{3} \mathrm{He}$ signal, we did not follow a rigid numerical procedure here due to fluctuations in background and uncertainties introduced by small numbers of counts in some cases. Rather each histogram is examined to see if a ${ }^{3} \mathrm{He}$ peak is apparent; if it is, a finite ${ }^{3} \mathrm{He} /{ }^{4} \mathrm{He}$ ratio is listed for that event. In practice this worked out to be a requirement that the net number of ${ }^{3} \mathrm{He}$ counts is approximately $2 \sigma$ or more above zero. If there is no plausible ${ }^{3} \mathrm{He}$ peak, a $1 \sigma$ upper limit is given. There are several events that have possible ${ }^{3} \mathrm{He}$ peaks but for which we report upper limits. All of the events with finite ${ }^{3} \mathrm{He} /{ }^{4} \mathrm{He}$ ratios have reasonable peaks; thus, the fraction of events in Table 2 with finite ${ }^{3} \mathrm{He}$ should be treated as a lower limit. Two examples (events 48 and 58) are shown in Figure 3, with the right panel showing an example of an event just above background. The $0.5-2.0 \mathrm{MeV}$ nucleon ${ }^{-1}{ }^{3} \mathrm{He} /{ }^{4} \mathrm{He}$ ratio (hereafter the $\sim 1 \mathrm{MeV}$ nucleon ${ }^{-13} \mathrm{He} /{ }^{4} \mathrm{He}$ ratio) in these events is respectively enhanced by factors of $\sim 30$ and $\sim 2$ over the solar wind value.

Of the 64 events in our survey, we found that 29 events (about $46 \%$ ) have finite and resolved ${ }^{3} \mathrm{He}$ peaks, with the ${ }^{3} \mathrm{He} /{ }^{4} \mathrm{He}$ ratio being enhanced between factors of $\sim 2-150$ over the slow solar wind value of $\sim 4.025 \times 10^{-4}$ (Gloeckler \& Geiss 1998). It is fair to ask if a factor of $\sim 2$ enhancement over the solar wind is significant or might be due to fluctuations in the solar wind ${ }^{3} \mathrm{He}$ abundance. Ho et al. (2000) reported several cases of solar wind ${ }^{3} \mathrm{He} /{ }^{4} \mathrm{He}$ enhancements of $\sim 4-10$ associated with CMEs; however, all but one of these were for relatively short periods of a few hours or less, and none of the periods found by Ho et al. is in the events studied in this paper. Although observations of solar wind ${ }^{3} \mathrm{He} /{ }^{4} \mathrm{He}$ averaged over multiday intervals such as those in Table 2 generally show little variation (Bodmer et al. 1995), if we take a conservative approach and count only those events with a factor of 4 enhancement as significant, then 23 events (36\%) in the survey meet this criterion.

\subsection{Heavy-Ion Abundances in Individual LSEP Events}

Table 2 also lists the heavy-ion abundances for individual LSEP events obtained by dividing the total fluence measured for each species by the $\mathrm{O}$ fluence in the $0.32-0.45 \mathrm{MeV}^{\text {nucleon }}{ }^{-1}$ energy range during the corresponding sampling intervals. Hereafter, we refer to these ratios as the $\sim 0.38 \mathrm{MeV}_{\text {nucleon }}{ }^{-1}$ fluence-integrated abundances. The error limits in the $\mathrm{C}-\mathrm{Fe}$ abundances are obtained by adding the statistical uncertainty in quadrature with an estimated systematic uncertainty of $\pm 2 \%$ that occurs due to changes in the average heavy-ion detection efficiency for ULEIS over the survey period (also see Desai et al. 2003).

For ${ }^{4} \mathrm{He}$, however, the detection efficiency of ULEIS decreased significantly as the instrument's microchannel plates aged due to radiation exposure during the period 1997 November-1999 April. These gain decreases were compensated by raising the high-voltage bias on the plates from time to time, causing large discontinuous increases in the microchannel plate gains, and thereby in the He efficiency. During this initial portion, covering the first eight events of the survey, the ${ }^{4} \mathrm{He}$ efficiency near $\sim 0.4 \mathrm{MeV}$ nucleon $^{-1}$ varied between $4 \%$ and $39 \%$ with a mean $\mu \sim 0.15$ and a standard deviation $\sigma \sim 0.104$. This yields an estimated systematic uncertainty $(\sigma / \mu)$ due to changes in the He detection efficiency of $\sim 69 \%$ over this period. Similar considerations for the rest of the survey period indicated that the $\mathrm{He}$ detection efficiency varied between $3 \%$ and $24 \%$ and had a mean $\mu \sim 0.1$ and standard deviation $\sigma \sim 0.033$. This yields an estimated systematic uncertainty of $\sim 31 \%$ for the survey period covering events $9-64$. The error limits in the ${ }^{4} \mathrm{He} / \mathrm{O}$ ratios listed in Table 2 are obtained by adding the statistical uncertainty in quadrature with estimated systematic uncertainties of $\sim 69 \%$ for events $1-8$ and $\sim 31 \%$ for the remainder of the events.

\subsection{Effects of Temporal Variations in Individual Events}

Rigidity-dependent transport processes can produce systematic temporal variations by up to a factor of $\sim 10$ in the Fe/O ratio during the onsets of some LSEP events (e.g., Tylka et al. 1999) or in the decay (e.g., Sollitt et al. 2003). In order to examine the effects of such variations on the fluence-integrated abundances listed in Table 2, we computed $\mathrm{Fe} / \mathrm{O}$ ratios on an hourly basis during each event and then calculated their unweighted mean value. We then compared these hourly averaged $\mathrm{Fe} / \mathrm{O}$ ratios with the event-integrated fluence ratios listed in Table 2. We found that most events exhibit a one-to-one correspondence. Although for some events, the hourly averaged $\mathrm{Fe} / \mathrm{O}$ ratio is significantly larger (more than $\sim 40 \%$ ) than the fluence-integrated ratio. 
TABLE 3

Mean Heavy-Ion Abundances for LSEP Events and Other Populations

\begin{tabular}{|c|c|c|c|c|c|c|c|c|c|c|}
\hline $\begin{array}{l}\text { Element }( \\
\text { (1) }\end{array}$ & $\begin{array}{c}\operatorname{LSEPs}^{\mathrm{a}} \\
0.38 \mathrm{MeV} \text { nucleon }^{-1} \text { ) } \\
\text { (2) }\end{array}$ & $\begin{array}{c}\text { Pre-Event }^{\mathrm{a}} \\
\left(\sim 0.38 \mathrm{MeV} \text { nucleon }^{-1}\right) \\
\text { (3) }\end{array}$ & $\begin{array}{c}\text { Slow } \mathrm{SW}^{\mathrm{b}, \mathrm{c}} \\
(\sim 1 \mathrm{keV} \text { nucleon } \\
\\
(4)\end{array}$ & $\begin{array}{c}\text { Fast } \mathrm{SW}^{\mathrm{b}, \mathrm{c}} \\
\left(\sim 2 \mathrm{keV} \text { nucleon }^{-1}\right) \\
(5)\end{array}$ & $\begin{array}{c}\text { IP Shocks }^{\mathrm{d}} \\
\left(\sim 0.75 \mathrm{MeV} \text { nucleon }^{-1}\right) \\
(6)\end{array}$ & $\begin{array}{c}\text { Gradual SEPs }{ }^{\mathrm{e}} \\
\left(5-12 \mathrm{MeV} \text { nucleon }^{-1}\right) \\
\text { (7) }\end{array}$ & $\begin{array}{c}\text { Impulsive SEPs }^{\mathrm{f}} \\
\left(0.385 \mathrm{MeV} \text { nucleon }^{-1}\right) \\
(8)\end{array}$ & $\begin{array}{c}\text { Photosphere } \\
\text { (9) }\end{array}$ & $\begin{array}{c}\text { Corona }^{\mathrm{h}} \\
\left(\sim 1.4 \times 10^{6} \mathrm{~K}\right) \\
(10)\end{array}$ & $\begin{array}{c}\text { CIRs }^{\mathrm{i}} \\
\left(\sim 1 \mathrm{MeV} \text { nucleon }^{-1}\right) \\
(11)\end{array}$ \\
\hline${ }^{4} \mathrm{He} . . . .$. & $75.0 \pm 23.6$ & $103.3 \pm 32.6$ & $95.9 \pm 28.8$ & $72.7 \pm 21.8$ & $44.4 \pm 14.4$ & $57 \pm 3$ & $54 \pm 14$ & $162 \pm 14$ & $126 \pm 11$ & $211 \pm 17$ \\
\hline С.............. & $0.361 \pm 0.012$ & $0.387 \pm 0.014$ & $0.670 \pm 0.067$ & $0.683 \pm 0.068$ & $0.368 \pm 0.004$ & $0.465 \pm 0.009$ & $0.322 \pm 0.003$ & $0.501 \pm 0.058$ & $0.490 \pm 0.056$ & $0.94 \pm 0.07$ \\
\hline $\mathrm{N} \ldots \ldots \ldots \ldots$ & $0.119 \pm 0.003$ & $0.130 \pm 0.005$ & $0.069 \pm 0.021$ & $0.111 \pm 0.033$ & $0.142 \pm 0.002$ & $0.124 \pm 0.003$ & $0.129 \pm 0.002$ & $0.138 \pm 0.022$ & $0.123 \pm 0.020$ & $0.15 \pm 0.04$ \\
\hline O................ & $\equiv 1.0 \pm 0.02$ & $\equiv 1.0 \pm 0.02$ & $\equiv 1$ & $\equiv 1$ & $\equiv 1$ & $\equiv 1$ & $\equiv 1.0 \pm 0.006$ & $\equiv 1.0 \pm 0.161$ & $\equiv 1.0 \pm 0.161$ & $\equiv 1$ \\
\hline $\mathrm{Ne} \ldots \ldots \ldots .$. & $0.152 \pm 0.005$ & $0.183 \pm 0.009$ & $0.091 \pm 0.027$ & $0.082 \pm 0.025$ & $0.172 \pm 0.003$ & $0.152 \pm 0.004$ & $0.261 \pm 0.003$ & $0.151 \pm 0.021$ & $0.191 \pm 0.026$ & $0.17 \pm 0.02$ \\
\hline $\mathrm{Mg} \ldots \ldots$. & $0.229 \pm 0.007$ & $0.239 \pm 0.012$ & $0.147 \pm 0.030$ & $0.105 \pm 0.021$ & $0.243 \pm 0.004$ & $0.196 \pm 0.004$ & $0.370 \pm 0.003$ & $0.072 \pm 0.009$ & $0.224 \pm 0.026$ & $0.10 \pm 0.02$ \\
\hline $\mathrm{Si} \ldots \ldots \ldots$. & $0.235 \pm 0.011$ & $0.226 \pm 0.011$ & $0.167 \pm 0.034$ & $0.115 \pm 0.023$ & $0.213 \pm 0.003$ & $0.152 \pm 0.004$ & $0.409 \pm 0.004$ & $0.071 \pm 0.007$ & $0.214 \pm 0.022$ & $0.08 \pm 0.01$ \\
\hline S ........... & $0.059 \pm 0.004$ & $0.057 \pm 0.004$ & $0.049 \pm 0.010$ & $0.056 \pm 0.011$ & $0.050 \pm 0.001$ & $0.032 \pm 0.001$ & $0.118 \pm 0.015$ & $0.032 \pm 0.008$ & $0.032 \pm 0.008$ & $0.03 \pm 0.01$ \\
\hline $\mathrm{Ca} \ldots \ldots \ldots .$. & $0.022 \pm 0.002$ & $0.025 \pm 0.003$ & $0.017 \pm 0.003$ & $0.0053 \pm 0.0014$ & $0.022 \pm 0.002$ & $0.011 \pm 0.001$ & $0.060 \pm 0.003$ & $0.005 \pm 0.0001$ & $10.013 \pm 0.0002$ & \\
\hline $\mathrm{Fe} . . . \ldots \ldots . .$. & $0.404 \pm 0.047$ & $0.359 \pm 0.044$ & $0.120 \pm 0.024$ & $0.092 \pm 0.018$ & $0.236 \pm 0.010$ & $0.134 \pm 0.004$ & $0.950 \pm 0.005$ & $0.061 \pm 0.006$ & $0.186 \pm 0.017$ & $0.10 \pm 0.01$ \\
\hline
\end{tabular}

${ }^{\text {a }}$ This work, based on 64 LSEP events observed with ACE ULEIS; ${ }^{4} \mathrm{He}$ abundances are averaged over events 9-64.

b See text for discussion of uncertainties in solar wind measurements (von Steiger et al. 2000).

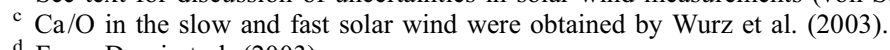

d From Desai et al. (2003).

e From Reames (1995a).

${ }^{\mathrm{f}}$ From Mason et al. (2004).

${ }^{\mathrm{g}}$ From Lodders (2003).

${ }^{\text {h }}$ From Feldman \& Widing (2003).

i From Mason et al. (1997). 

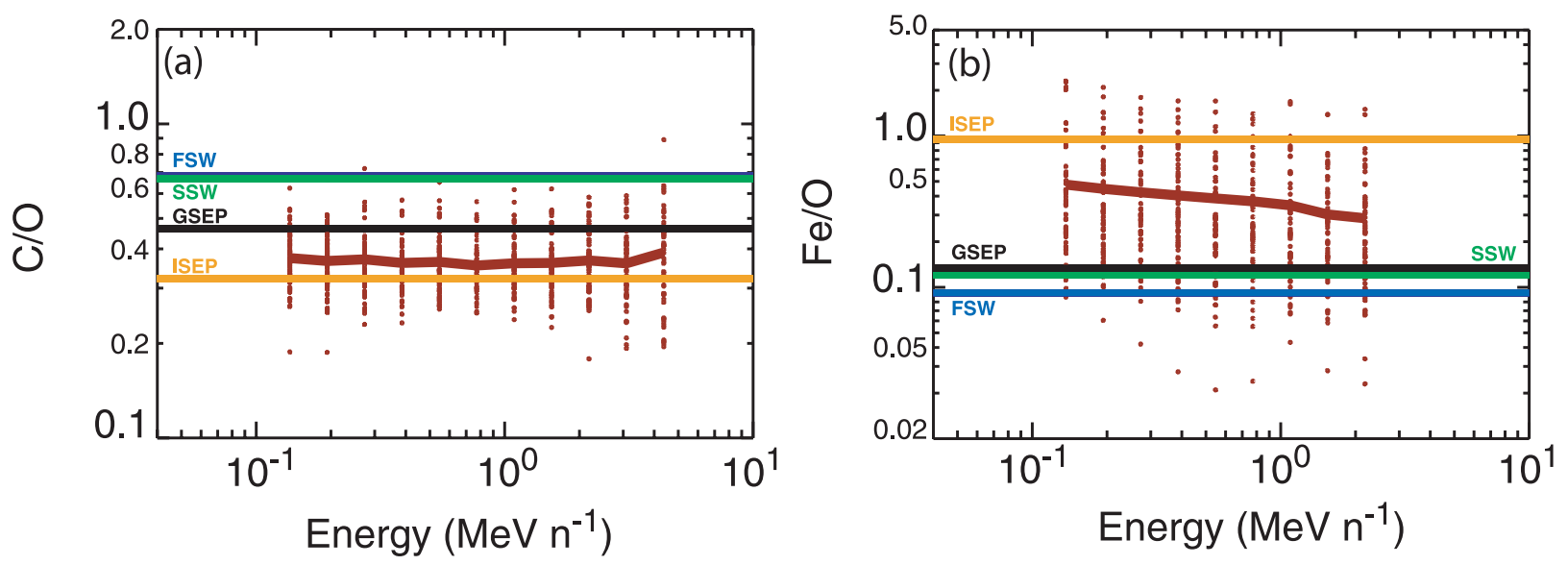

FIG. 4.- (a) C/O and (b) Fe/O ratios vs. energy for all 64 LSEP events. Each data point represents the abundance ratio within a specific energy range for one event.

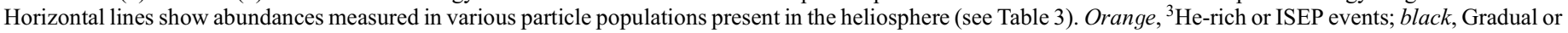
GSEP events; blue, fast solar wind (FSW); and green, slow solar wind (SSW).

In order to examine the effects of our choice of the averaging technique on the deduced abundances, we then used the fluenceintegrated and hourly averaged $\mathrm{Fe} / \mathrm{O}$ ratios during individual events to compute separate unweighted averages for the entire survey. In general, the survey-averaged $\mathrm{Fe} / \mathrm{O}$ ratios calculated from the fluences were about $\sim 20 \%$ lower than those computed from the hourly averages. Specifically, at $\sim 0.38 \mathrm{MeV}_{\text {nucleon }}{ }^{-1}$, the average $\mathrm{Fe} / \mathrm{O}$ obtained from the fluences is $0.404 \pm 0.047$, while the average value obtained using the hourly averaged $\mathrm{Fe} / \mathrm{O}$ is $0.49 \pm 0.057$. These differences are probably systematic and most likely occur due to temporal variations during the onsets, as discussed earlier. Nonetheless, the survey-averaged values from the two methods are well within the $1 \sigma$ uncertainties, implying that the systematic $M / Q$-dependent temporal variations in the $\mathrm{Fe} / \mathrm{O}$ ratios that typically occur during the onsets of LSEP events have a relatively minor influence on both the fluence-integrated abundances listed in Table 2 and the unweighted averages calculated from them.

\subsection{Survey-Averaged Heavy-Ion Abundances}

Table 3 lists the $\sim 0.38 \mathrm{MeV}$ nucleon $^{-1}$ unweighted average abundances for the LSEP survey (col. [2]) computed from the individual fluence-integrated abundances listed in Table 2. For ${ }^{4} \mathrm{He}$, we only used events 9-64 because the estimated systematic uncertainty due to changes in the average He detection efficiency during the time period covering events $1-8$ was $\sim 69 \%$. The uncertainties in the survey-averaged abundances in Table 3 are calculated by adding the standard error of the mean in quadrature with estimated systematic uncertainties of $31 \%$ for ${ }^{4} \mathrm{He}$ and $2 \%$ for $\mathrm{C}-\mathrm{Fe}$.

Table 3 also compares the survey-averaged LSEP abundances with those measured in "pre-event" intervals (col. [3]) and in various populations in the inner solar system. The pre-event interval for a given LSEP event is defined as the 8 day interval preceding that event. Columns (4) and (5) provide solar wind average abundances based on daily values for 300 day intervals (von Steiger et al. 2000). The uncertainties in the solar wind values are calculated as follows: we divided the $1 \sigma$ dispersion values given in Table 1 of von Steiger et al. (2000) by $300^{1 / 2}$ and added them in quadrature with the stated maximum possible systematic uncertainties of $\sim 10 \%$ for $\mathrm{C}$ and $\mathrm{O}, \sim 30 \%$ for ${ }^{4} \mathrm{He}, \mathrm{N}$, and $\mathrm{Ne}$, and $\sim 20 \%$ for the other elements (see also Mewaldt et al. 2002;
Desai et al. 2003). Column (6) provides the $\sim 0.75 \mathrm{MeV}$ nucleon ${ }^{-1}$ mean abundances in 72 CME-driven IP shocks at $A C E$ (from Desai et al. 2003). The mean abundances in gradual SEP events at $5-12 \mathrm{MeV}$ nucleon ${ }^{-1}$ (Reames 1995a) and in ${ }^{3} \mathrm{He}$-rich SEP events (Mason et al. 2004) are given in columns (7) and (8), respectively. Columns (9) and (10) provide abundances measured in the solar photosphere (Lodders 2003) and in the quiet ( 1.4 MK) solar corona (Feldman \& Widing 2003), respectively. Column (11) lists the mean abundances in corotating interaction regions (CIRs; Mason et al. 1997).

\section{PROPERTIES OF $\mathrm{Fe} / \mathrm{O}$}

\subsection{Energy Dependence of Mean $\mathrm{C} / \mathrm{O}$ and $\mathrm{Fe} / \mathrm{O}$ Ratios}

In order to examine the energy dependence and the event-toevent variability of the heavy-ion abundances, we calculated the fluence-integrated abundances of all species in several energy bands with respect to $\mathrm{O}$. Figure $4 a$ shows the $\mathrm{C} / \mathrm{O}$ ratios between 0.11 and $5.12 \mathrm{MeV}$ nucleon ${ }^{-1}$, and Figure $4 b$ shows the $\mathrm{Fe} / \mathrm{O}$ ratios between 0.11 and $2.56 \mathrm{MeV}$ nucleon $^{-1}$ separated into 11 and 9 energy intervals, respectively. Values with statistical uncertainties greater than $35 \%$ are excluded from this figure and from the subsequent analysis. Brown curves represent the mean abundances versus energy. Average $\mathrm{C} / \mathrm{O}$ and $\mathrm{Fe} / \mathrm{O}$ ratios measured in ${ }^{3} \mathrm{He}$-rich and gradual SEP events, and fast and slow solar wind from Table 3 are also shown in the figure.

The scatter of the data points along the $y$-axis shows the eventto-event variations at a given energy, while the points along the $x$-axis show their energy dependence. Figure $4 a$ shows that most of the data points and the mean $\mathrm{C} / \mathrm{O}$ ratios at all energies are substantially lower than those measured in the fast and slow solar wind, but similar to those measured in gradual and ${ }^{3} \mathrm{He}$-rich SEP events. Only 10 of the $690 \mathrm{C} / \mathrm{O}$ ratios (i.e., less than $2 \%$ ) are within the error limits of the average fast and slow solar wind values. Figure $4 b$ shows that the $\mathrm{Fe} / \mathrm{O}$ ratios are more variable and exhibit a relatively broader distribution than the $\mathrm{C} / \mathrm{O}$ ratios. Also, the mean $\mathrm{Fe} / \mathrm{O}$ ratio decreases by about a factor of 2 with increasing energy, while the mean $\mathrm{C} / \mathrm{O}$ ratios remain constant over the ULEIS energy range. Finally, the mean $\mathrm{Fe} / \mathrm{O}$ ratios below $\sim 0.5 \mathrm{MeV}$ nucleon ${ }^{-1}$ are about a factor of $\sim 5$ greater than those measured in the fast and slow solar wind and in gradual SEP events at 5-12 MeV nucleon ${ }^{-1}$, but a factor of 2 lower than that measured in ${ }^{3} \mathrm{He}$-rich SEP events. 

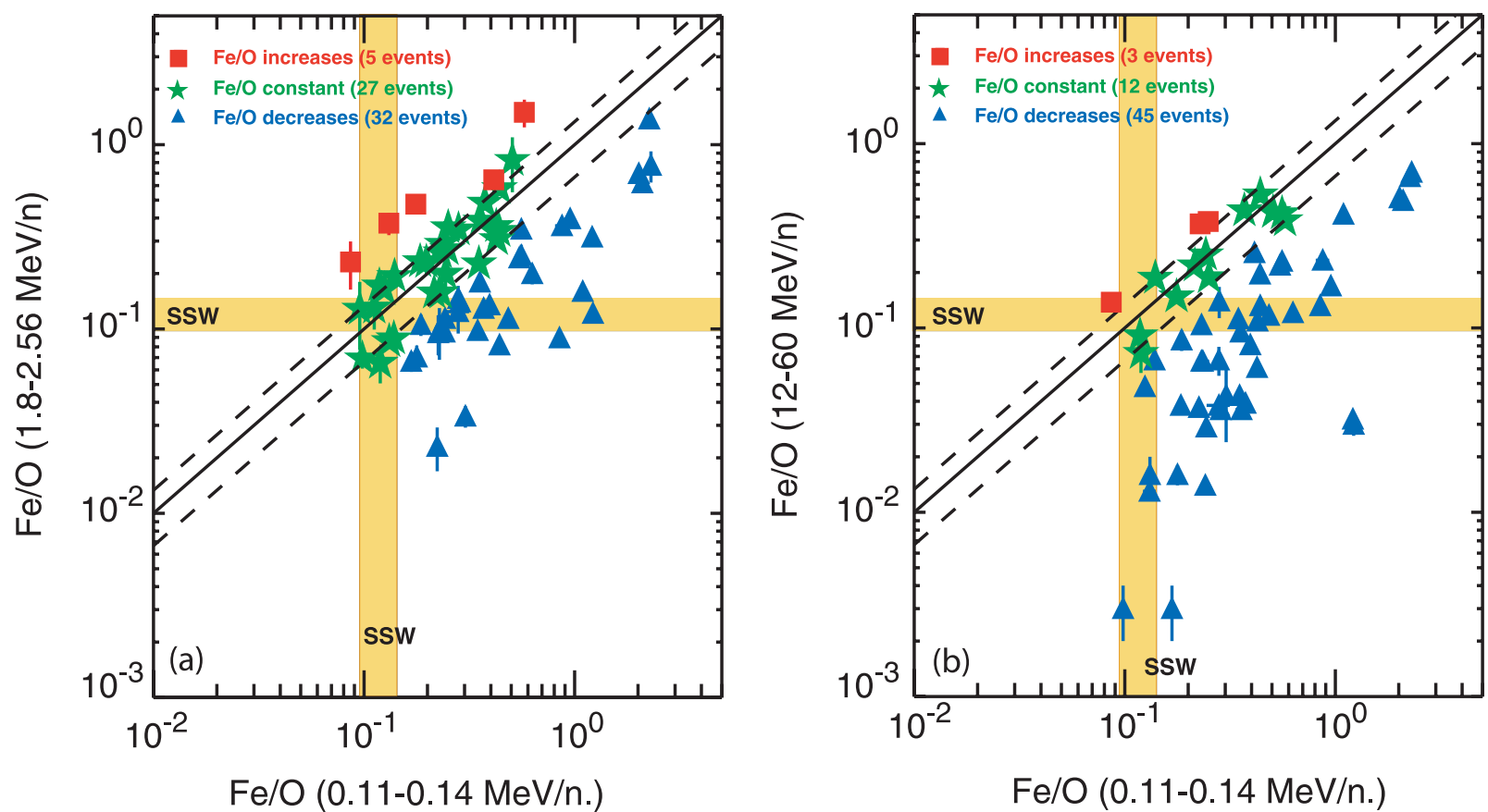

FIg. 5.-Energy-dependent behavior of Fe/O ratio in LSEP events. The Fe/O ratio at $0.11-0.14 \mathrm{MeV}$ nucleon $^{-1}$ is plotted vs. that at (a) $1.8-2.56 \mathrm{MeV}$ nucleon ${ }^{-1}$ from ULEIS and $(b) 12-60 \mathrm{MeV}$ nucleon ${ }^{-1}$ from SIS. Dashed lines identify events where Fe/O remained constant with energy within $\pm 33 \%$. Red squares, Fe/O increases with increasing energy; green asterisks, $\mathrm{Fe} / \mathrm{O}$ remains constant with energy; blue triangles, $\mathrm{Fe} / \mathrm{O}$ decreases with increasing energy; and yellow bands: error limits of the average $\mathrm{Fe} / \mathrm{O}$ ratio measured in the slow solar wind.

We also examined the energy dependence and event-to-event variations of the ${ }^{4} \mathrm{He}, \mathrm{N}, \mathrm{Ne}, \mathrm{Mg}, \mathrm{Si}, \mathrm{S}$, and $\mathrm{Ca}$ abundances. The distributions of ${ }^{4} \mathrm{He}, \mathrm{N}, \mathrm{Ne}, \mathrm{Mg}$, and $\mathrm{Si}$ abundances over the ULEIS energy range resemble that of the $\mathrm{C} / \mathrm{O}$ ratio shown in Figure $4 a$. The $\mathrm{Ca}$ and $\mathrm{S}$ abundances exhibit broader distributions than that of the $\mathrm{C} / \mathrm{O}$ ratio, but narrower than that of the $\mathrm{Fe} / \mathrm{O}$ ratio. The $\mathrm{Ca}$ and $\mathrm{S}$ abundances also exhibit substantial event-toevent variations; however, their means do not exhibit any systematic energy dependence, as seen in the case of $\mathrm{Fe}$.

\subsection{Energy Dependence of $\mathrm{Fe} / \mathrm{O}$ in Individual LSEP Events}

In order to examine the effects of acceleration processes in individual LSEP events, we plot in Figure 5 the $\mathrm{Fe} / \mathrm{O}$ ratio measured at $0.11-0.14 \mathrm{MeV}$ nucleon $^{-1}$ versus that measured at $(a) 1.8$ $2.56 \mathrm{MeV}$ nucleon $^{-1}$ by ULEIS and at $(b) 12-60 \mathrm{MeV}$ nucleon $^{-1}$ by SIS. This figure clearly shows that the $\mathrm{Fe} / \mathrm{O}$ ratio decreases with increasing energy in most of the LSEP events in our survey. Decreases in the SEP Fe/O ratios at higher energies have also been reported by a number of previous studies (e.g., Mazur et al. 1992; Tylka et al. 2005; Cohen et al. 2005). Similar spectral properties were also observed in individual CME-driven IP shocks at $A C E$ (see Desai et al. 2003, 2004). Furthermore, the low-energy Fe/O ratio in 55 of the 64 LSEP events in our survey is enhanced when compared with the upper error limit of the average value measured in the slow solar wind.

\subsection{Comparison with Previous Surveys}

Figure 6 compares the mean $\mathrm{Fe} / \mathrm{O}$ ratios measured by ULEIS and SIS for the 64 LSEP events in this survey with those obtained by previous studies, plotted as a function of energy. The uncertainty in the $A C E$ data is the standard error in the mean and is calculated from the $1 \sigma$ standard deviation that results from the event-to-event variations seen along the $y$-axis in Figure $4 b$. It is worth noting that the $\mathrm{Fe} / \mathrm{O}$ ratios measured by ULEIS and SIS represent average values for the same sample of 64 events, and consequently the $A C E$ data points are not independent of each other. In contrast, the average $\mathrm{Fe} / \mathrm{O}$ ratios in prior surveys were based on different sets of SEP events and are therefore independent measurements. We note the following: (1) The average $\mathrm{Fe} / \mathrm{O}$ ratio obtained in earlier surveys in the $\sim 1-50 \mathrm{MeV}$ nucleon ${ }^{-1}$ energy range was $\sim 0.12$ and is lower than the average value at

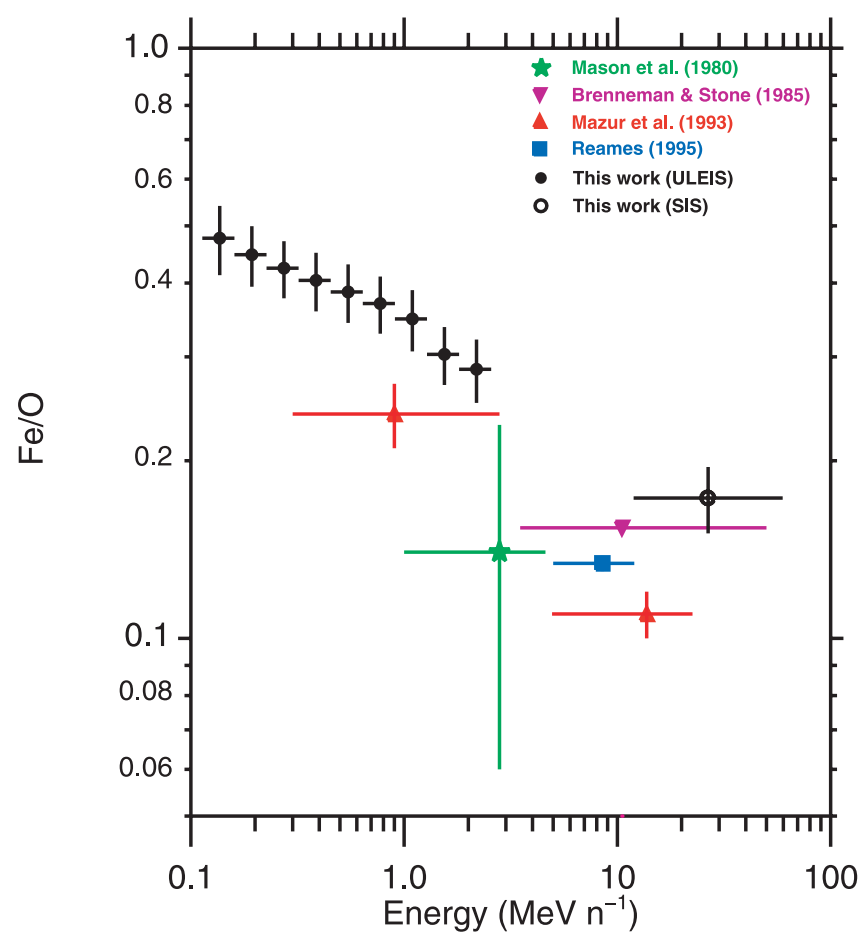

FIG. 6.- Average $\mathrm{Fe} / \mathrm{O}$ ratios measured at $A C E$ in this survey compared with those measured in prior surveys, as a function of energy. 

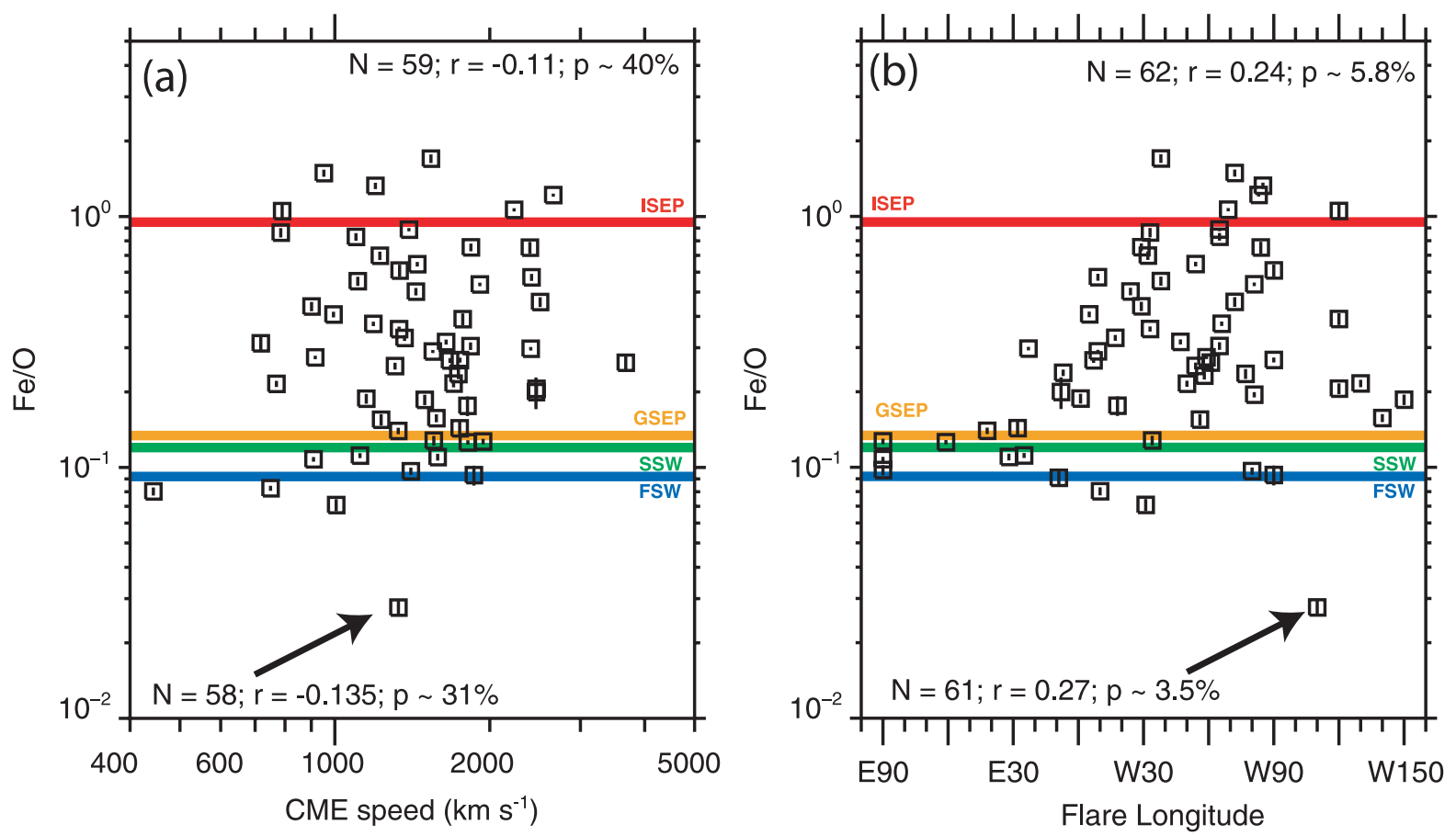

Fig. 7.- The $0.38 \mathrm{MeV}$ nucleon ${ }^{-1} \mathrm{Fe} / \mathrm{O}$ ratios vs. (a) CME speed (kilometers per second) and (b) flare longitude. In both cases, the correlation coefficient $r$ is also calculated after omitting event 45 (marked by arrow) from the regression fit.

$\sim 12-60 \mathrm{MeV}^{\text {nucleon }}{ }^{-1}$ obtained by SIS. (2) The mean Fe/O ratios obtained by ULEIS below $\sim 2 \mathrm{MeV}$ nucleon $^{-1}$ are significantly higher when compared with those obtained at higher energies and between $\sim 0.3$ and $3 \mathrm{MeV}$ nucleon ${ }^{-1}$ energy by Mazur et al. (1993).

\subsection{Fe/O Versus CME Speed and Flare Longitude}

Figure 7 investigates the relationship between the $\sim 0.38 \mathrm{MeV}$ nucleon $^{-1} \mathrm{Fe} / \mathrm{O}$ ratio from Table 2 and $(a)$ the CME speed (kilometers per second) and $(b)$ the flare longitude from Table 1 . This figure demonstrates the lack of a significant correlation between the low-energy $\mathrm{Fe} / \mathrm{O}$ ratio and both the $\mathrm{CME}$ speed and the flare longitude. Note that the statistical significance in both cases is not affected by the outlier event 45 with low Fe/O at W110 (marked by arrow). It is striking that the $\mathrm{Fe} / \mathrm{O}$ ratios in all six LSEP events associated with the eastern hemisphere flares/CMEs $(>E 30)$ are $\sim 0.1$. However, this sample is too small to allow firmer conclusions about reported associations between eastern hemisphere events and lower $\mathrm{Fe} / \mathrm{O}$ ratios above $\sim 25 \mathrm{MeV}$ nucleon ${ }^{-1}$ (e.g., see Cane et al. 2003).

\subsection{Fe/O Versus Fluence}

Figure 8 investigates the relationship between the $\sim 0.38 \mathrm{MeV}$ nucleon $^{-1} \mathrm{Fe} / \mathrm{O}$ ratio and the size of the LSEP event by plotting the $\mathrm{Fe}$ versus the $\mathrm{O}$ fluences during the event. Most events have $\mathrm{Fe} / \mathrm{O}$ ratios between 0.1 and 1.0, as can also be seen from Figure $4 b$. Furthermore, events with high and low $\mathrm{Fe} / \mathrm{O}$ ratios are associated with a wide range of $\mathrm{Fe}$ and $\mathrm{O}$ fluences, with event 57 having the largest $\mathrm{Fe}$ and $\mathrm{O}$ fluences and a relatively high $\mathrm{Fe} / \mathrm{O}$ of $\sim 1.0$. In general, the figure shows that the low-energy $\mathrm{Fe} / \mathrm{O}$ ratio is independent of the size of the LSEP event (see also Mewaldt et al. 2006).

\section{6. $\mathrm{Fe} / \mathrm{O}$ in LSEPs and Pre-Event Intervals}

In order to compare the heavy-ion abundances in LSEP events with those measured in the suprathermal ion population present in the IP medium near 1 AU (Mason 2000; Gloeckler 2003), we plot in Figure 9 the $1.8-2.56 \mathrm{MeV}$ nucleon $^{-1} \mathrm{Fe} / \mathrm{O}$ ratio in 46 LSEP events versus the $0.11-0.16 \mathrm{MeV}$ nucleon ${ }^{-1} \mathrm{Fe} / \mathrm{O}$ measured during the corresponding pre-event intervals. Eighteen events for which the pre-event interval included a prior LSEP

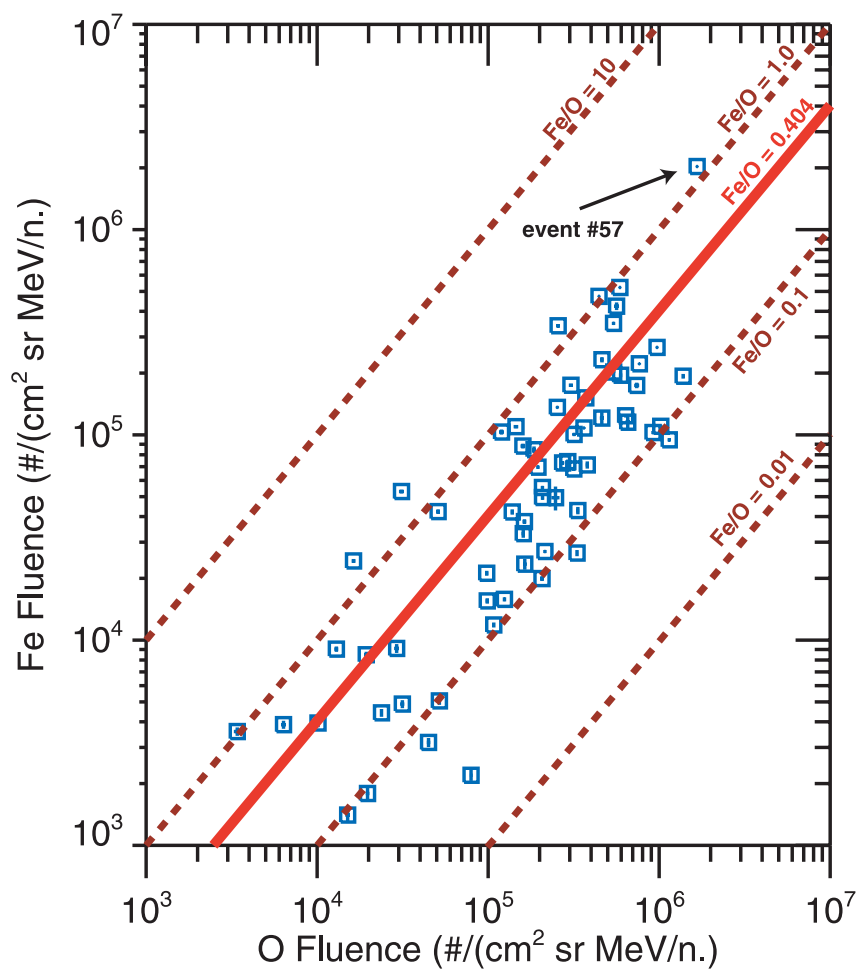

FIG. 8.-Fe fluence vs. O fluence at $0.38 \mathrm{MeV}$ nucleon $^{-1}$ : dashed lines, $\mathrm{Fe} / \mathrm{O}$ ratios of $0.01,0.1,1.0$, and 10.0 ; red line, average $\mathrm{Fe} / \mathrm{O}$ ratio obtained in this LSEP survey. 


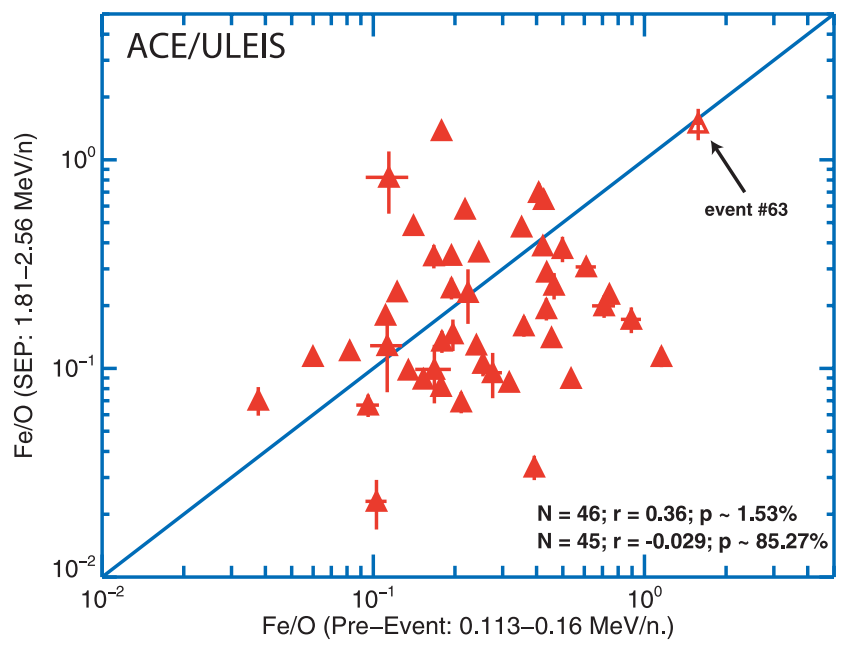

FIG. 9.- The 1.8-2.56 MeV nucleon ${ }^{-1} \mathrm{Fe} / \mathrm{O}$ in 46 LSEP events vs . 0.11$0.16 \mathrm{MeV}$ nucleon ${ }^{-1} \mathrm{Fe} / \mathrm{O}$ measured in pre-event intervals. Eighteen events for which the corresponding pre-event interval included a prior LSEP event are excluded. The correlation coefficient $r$ is calculated for 46 events and for 45 events by omitting event 63 (open triangle).

event are not included in the figure. The correlation coefficient ( $r=0.36$ ) for all 46 events has $\sim 1.53 \%$ chance of being from random populations. However, this probability depends largely on event 63 (open triangle), without which the correlation coefficient $r=-0.029$ has a very high probability $(p \sim 85 \%)$ that these two populations are unrelated. In summary, the data shown in Figure 9 do not support the notion that the Fe/O ratio in LSEP events is correlated with that measured in the suprathermal ion population present near $1 \mathrm{AU}$ (see also Mewaldt et al. 2006).

\section{4. ${ }^{3} \mathrm{He}$ ENHANCEMENTS}

\section{1. ${ }^{3} \mathrm{He} /{ }^{4} \mathrm{He}$ Ratio Versus $\mathrm{Fe} / \mathrm{O}$ Ratio}

Figure 10 shows a scatter plot of the ${ }^{3} \mathrm{He} /{ }^{4} \mathrm{He}$ versus the $\mathrm{Fe} / \mathrm{O}$ ratio at $\sim 1 \mathrm{MeV}$ nucleon ${ }^{-1}$ for the 29 LSEP events with finite ${ }^{3} \mathrm{He}$ from Table 2 . The correlation coefficient $(r=0.66)$ for all 29 events is statistically significant with a low probability ( $\sim 0.01 \%$ ) of resulting from random populations. However, this correlation depends largely on events 20 and 29 (open squares), without which the correlation coefficient $(r=0.026)$ has a high probability ( $p \sim 90 \%$ ) that these ratios may be uncorrelated. Although there is little justification for excluding these events from the regression fit, the scatter of the data points indicates that the $\mathrm{Fe} / \mathrm{O}$ and ${ }^{3} \mathrm{He} /{ }^{4} \mathrm{He}$ ratios in LSEP events are probably poorly correlated.

\subsection{Solar Activity Dependence}

In order to examine possible solar cycle dependences we divided the data set into five periods: (1) 1997 November-1999 December, 12 events; (2) 2000 January-2000 December, 10 events; (3) 2001 January-2001 December, 16 events; (4) 2002 January-2002 December, 13 events; and (5) 2003 January2005 January, 13 events. Using the appropriate quantities from Table 2, we calculated the mean $\mathrm{Fe} / \mathrm{O}$ ratios and the occurrence frequency of events with finite ${ }^{3} \mathrm{He}$ during each period. Figure 11 shows $(a)$ the mean $\mathrm{Fe} / \mathrm{O}$ ratio, $(b)$ the incidence of finite ${ }^{3} \mathrm{He}$ events, and $(c)$ the occurrence rates of X-ray flares (C-, M-, and $\mathrm{X}$-class) and sunspots, ${ }^{2}$ plotted versus time. The corresponding

\footnotetext{
${ }^{2}$ Both obtained from http://sec.noaa.gov/ftpmenu/indices/old indices.html.
}

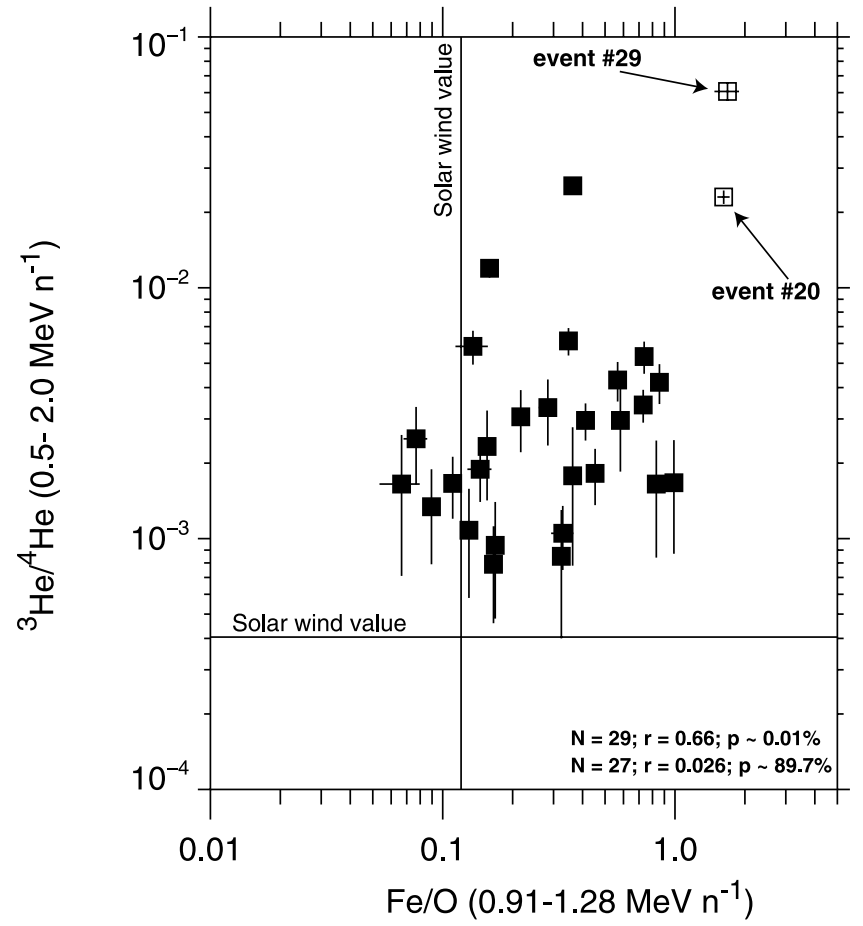

FIG. 10.-The $0.5-2.0 \mathrm{MeV}$ nucleon ${ }^{-1}{ }^{3} \mathrm{He} /{ }^{4} \mathrm{He}$ ratio vs. $0.91-1.28 \mathrm{MeV}$ nucleon $^{-1} \mathrm{Fe} / \mathrm{O}$ ratio in $29 \mathrm{LSEP}$ events. The correlation coefficient $r$ and its statistical significance $p$ are calculated for 29 events and for 27 events by omitting events 20 and 29 (open squares).

quantities measured for IP shock events from the survey of Desai et al. (2003) are also shown. The number of sunspots and flares are normalized to their corresponding maxima during each of the five periods.

The incidence of LSEP events with finite ${ }^{3} \mathrm{He}$ remains relatively constant between $\sim 40 \%$ and $50 \%$ for the first three subperiods between 1997 November and 2001 December and reaches a peak value of $\sim 60 \%$ during 2002 . The occurrence rate then drops by a factor of $2 \%$ to $\sim 25 \%$ between 2003 January and 2005 January. In contrast, the occurrence rate of ${ }^{3} \mathrm{He}$-rich IP shock events peaks in 1999 and drops in 2002. The mean Fe/O ratio in LSEP events shows no clear trend with solar activity and remains reasonably constant with values that fall between those measured in ${ }^{3} \mathrm{He}$-rich and gradual SEP events (also see Fig. 4). In contrast, the Fe/O ratio in IP shock events increases with solar activity between 1998 and 2002 .

\section{COMPARISON WITH HELIOSPHERIC ION POPULATIONS}

\subsection{LSEP Versus Solar Wind Abundances}

Figure $12 a$ shows the $0.32-45 \mathrm{MeV}$ nucleon $^{-1}$ average LSEP abundances normalized to those measured in the slow and fast solar wind plotted versus $M / Q$ (amu $\left.e^{-1}\right)$. The charge states are taken as those measured in the slow solar wind (from von Steiger et al. 1997). These are $\mathrm{He}^{2+}, \mathrm{C}^{5.38+}, \mathrm{N}^{5.47+}, \mathrm{O}^{6.05+}, \mathrm{Ne}^{7.97+}$, $\mathrm{Mg}^{9.5+}, \mathrm{Si}^{8.57+}, \mathrm{S}^{8.75+}, \mathrm{Ca}^{9.02+}$, and $\mathrm{Fe}^{9.84+}$. We note the following: (1) The LSEP abundances are poorly correlated with both the fast and slow solar wind values. (2) C, N, O, Ne, and $\mathrm{Mg}$ have similar $M / Q$ values but exhibit highly unsystematic enhancements and depletions relative to the solar wind values, with $\mathrm{C}$ in particular being depleted by about a factor of 2. (3) The abundances of $\mathrm{Si}, \mathrm{Ca}$, and $\mathrm{Fe}$ are enhanced relative to the solar wind values. Note that if the heavier ions (e.g., Fe) were stripped of 


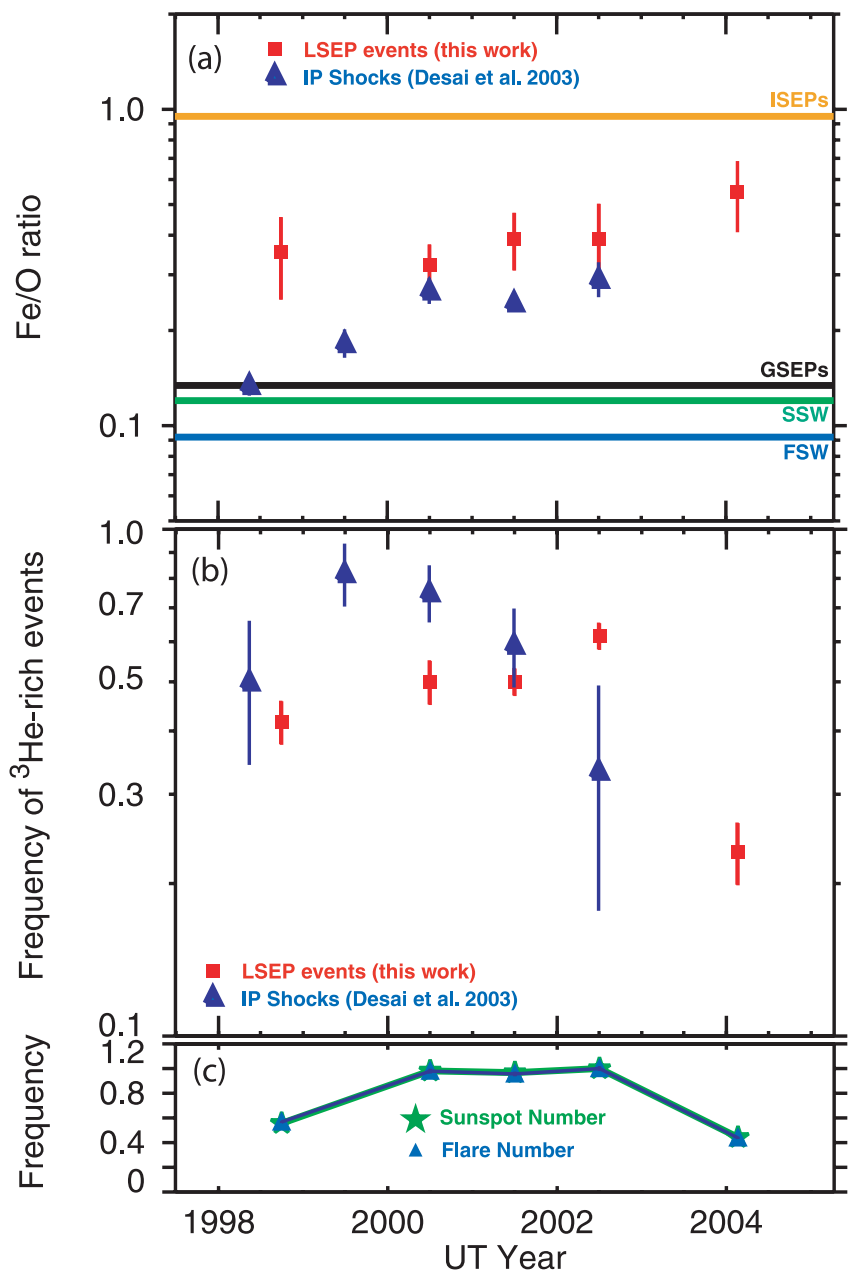

FIG. 11.- (a) Mean Fe/O ratio in IP shocks and LSEP events, $(b)$ frequency of IP shock events and LSEP events with finite ${ }^{3} \mathrm{He}$, and $(c)$ occurrence rates of $\mathrm{X}$-ray flares and sunspots vs. time. The IP shock data are obtained from Desai et al. (2003). The occurrence rates of X-ray flares and sunspots are normalized to their respective maxima.

more of their electrons, then their $M / Q$ ratios would be somewhat lower. In this case the data points for these ions would shift horizontally toward the left of the plot, but their abundances would still remain significantly enhanced when compared with the solar wind values.

Figure $12 b$ shows the average abundances for nine events with upper limits for the ${ }^{3} \mathrm{He} /{ }^{4} \mathrm{He}$ ratio and with $\mathrm{Fe} / \mathrm{O}$ ratios near $\sim 0.13 \mathrm{MeV}$ nucleon $^{-1}$ less than or equal to the average slow solar wind value of 0.12 plus an upper limit of 0.024 (see Table 3 ). These events correspond to events $2,7,8,10,14,40,44,54$, and 61 in Tables 1 and 2 and are most likely to have accelerated material with composition like the solar wind. However, Figure $12 b$ shows that the average abundances in these events when normalized to the corresponding solar wind values are uncorrelated with the $M / Q$ ratio and therefore are not related to solar wind elemental abundances in any simple manner.

\subsection{LSEP Versus Energetic Particle Abundances}

Figure 13 shows the average LSEP abundances normalized to those measured in ${ }^{3} \mathrm{He}$-rich SEP events, gradual SEP events at 5-12 MeV nucleon ${ }^{-1}$, CME-driven IP shock events, and preevent intervals, plotted versus $M / Q$. The charge states for various species are taken as the mean ionization states measured in gradual SEP events between 0.18 and $0.45 \mathrm{MeV}^{\text {nucleon }}{ }^{-1}$ (Klecker
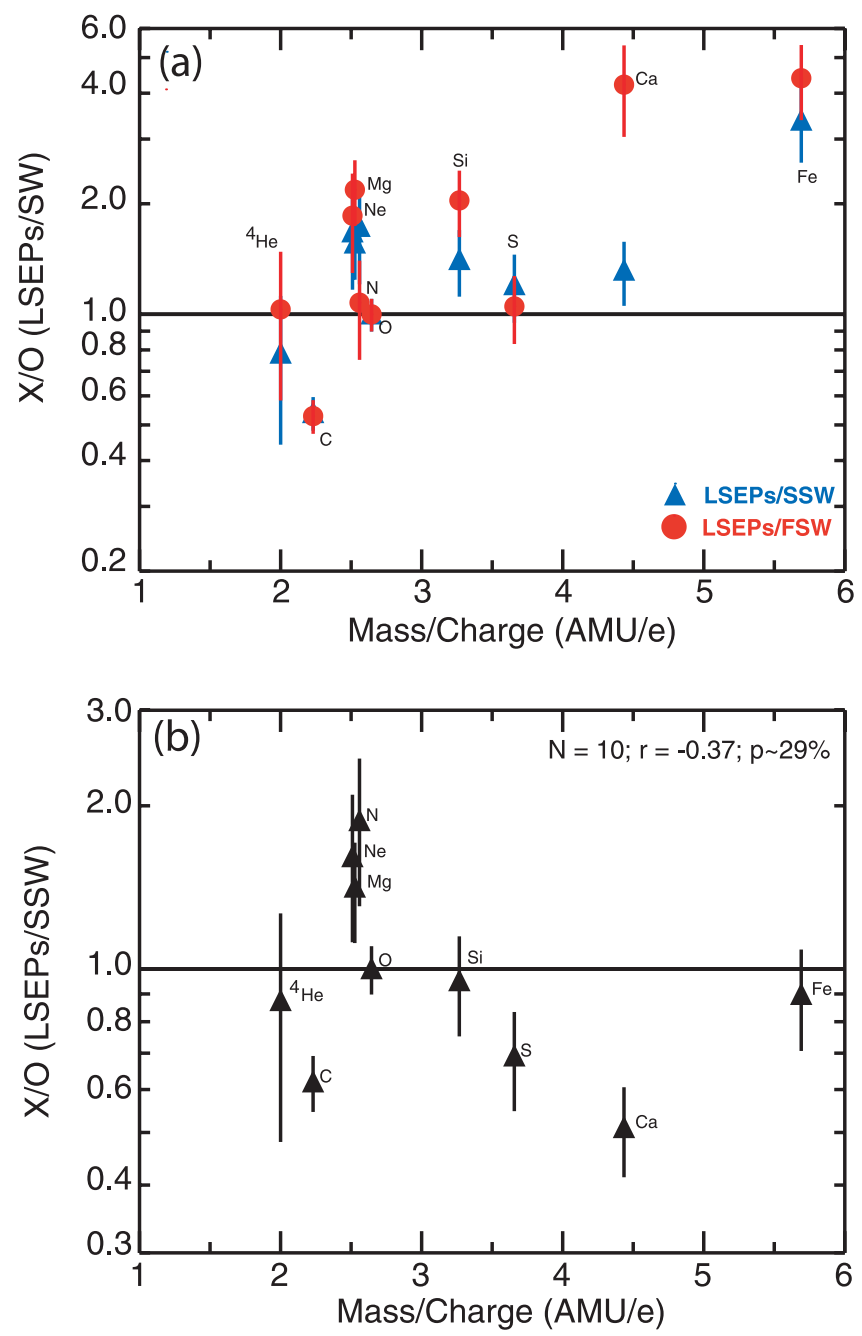

FIG. 12.-(a) Mean heavy-ion abundances in LSEP events relative to those measured in the fast and slow solar wind, normalized to oxygen and plotted vs. $M / Q$. (b) Same as (a), but for nine LSEP events with upper limits for the ${ }^{3} \mathrm{He} /{ }^{4} \mathrm{He}$ ratio and $\mathrm{Fe} / \mathrm{O}$ ratio less than 0.144 (see text for details).

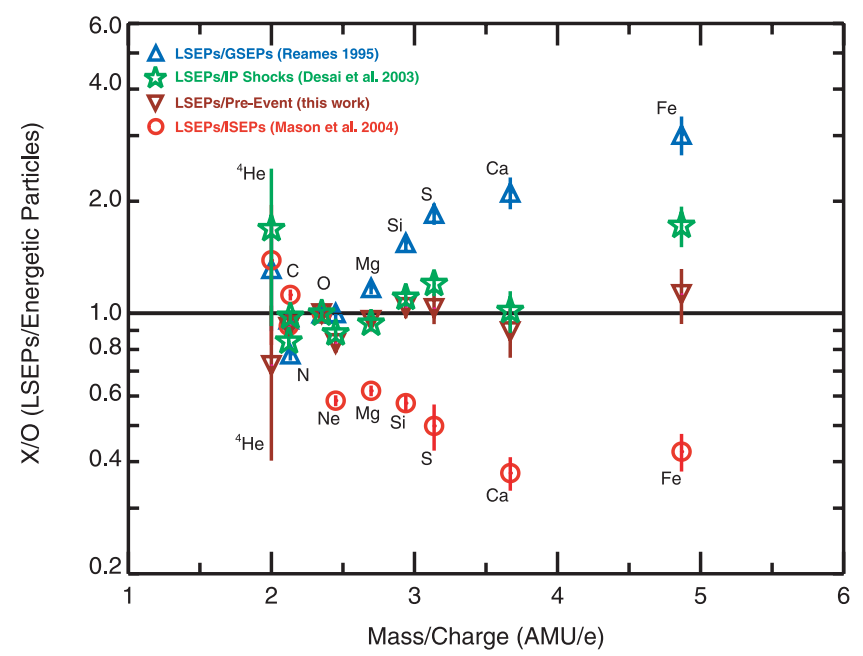

FIG. 13.-Mean heavy-ion abundances in LSEP events relative to those measured in various energetic particle populations given in Table 3, normalized to oxygen and plotted vs. $M / Q$ : blue triangles, gradual SEPs; green asterisks, IP shocks; downward-pointing-brown triangles, pre-event suprathermals; and red circles, impulsive SEPs. 

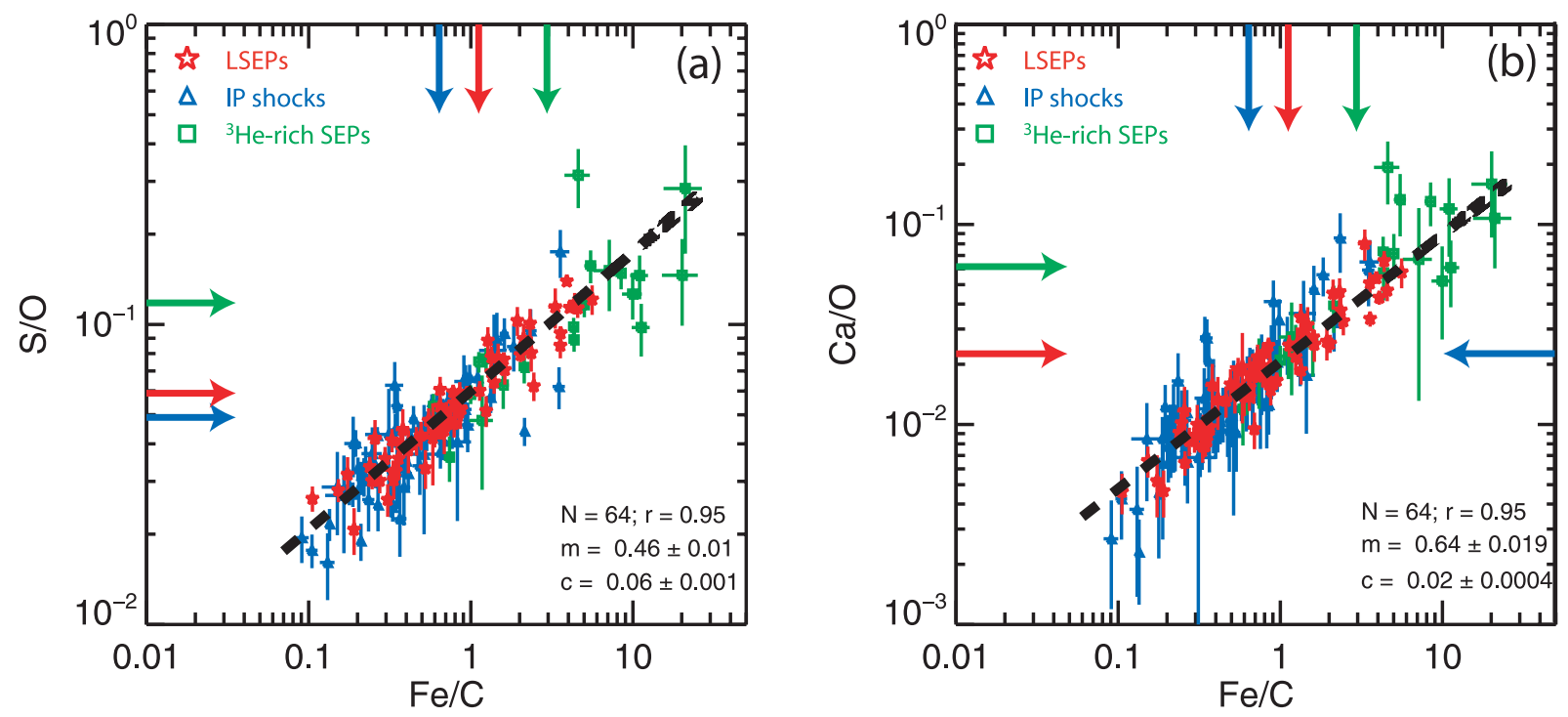

FIG. 14.- (a) $\mathrm{S} / \mathrm{O}$ and (b) Ca/O plotted vs. Fe/C at $0.38 \mathrm{MeV}$ nucleon $^{-1}$ for three different types of events: blue triangles, IP shock events of Desai et al. (2003); red asterisks, LSEP events in this survey, and green squares, ${ }^{3} \mathrm{He}$-rich SEP events of Mason et al. (2004). Colored arrows along the axes represent the respective average values given in Table 3. The dashed black line represents the linear fit to the LSEP data. The quantities $N$ and $r$ represent the number of LSEP events and the correlation coefficient, respectively. The quantities $m$ and $c$ represent the slopes and intercepts of the linear fits, respectively.

et al. 1999, 2000; Möbius et al. 1999, 2000). These are $\mathrm{He}^{2+}$, $\mathrm{C}^{5.6+}, \mathrm{N}^{6.6+}, \mathrm{O}^{6.8+}, \mathrm{Ne}^{8.2+}, \mathrm{Mg}^{8.9+}, \mathrm{Si}^{9.5+}, \mathrm{S}^{10.2+}, \mathrm{Ca}^{10.8+}$, and $\mathrm{Fe}^{11.6+}$. Several features are clearly evident: (1) The LSEP abundances are systematically enhanced and depleted with increasing $M / Q$ ratio when compared with those measured in gradual and ${ }^{3} \mathrm{He}-$ rich SEP events, respectively. (2) The LSEP abundances fall between those measured in ${ }^{3} \mathrm{He}$-rich and gradual SEP events (also see Figs. 4 and 11). (3) The LSEP abundances are generally similar to those measured in IP shocks; however, the $\mathrm{Fe} / \mathrm{O}$ ratio in LSEP events is enhanced by about a factor of 2. (4) On average, the LSEP abundances are remarkably similar to those measured in pre-event intervals; however, recall that the $\mathrm{Fe} / \mathrm{O}$ ratio in LSEP events and pre-event intervals are uncorrelated on a case-by-case basis (see Fig. 9). As in Figure 12, differences in the ionization states of the heavier ions do not affect the results.

\section{6. $M / Q$-DEPENDENT FRACTIONATION OF HEAVY-ION ABUNDANCES}

\subsection{Event-to-Event Enhancement Pattern}

Figure 14 examines the enhancement pattern of heavy-ion abundances in LSEP events and compares it with that seen in IP shock events of Desai et al. (2003) and ${ }^{3} \mathrm{He}-$ rich SEP events of Mason et al. (2004). Plotted here is $(a)$ the $\mathrm{S} / \mathrm{O}$ ratio and $(b)$ the $\mathrm{Ca} / \mathrm{O}$ ratio versus the $\mathrm{Fe} / \mathrm{C}$ ratio at $\sim 0.38 \mathrm{MeV}$ nucleon $^{-1}$ for the three types of events. The dashed line represents a linear fit to the LSEP data with slope $m$ and intercept $c$. The linear correlation coefficient $r$ in each case is also given. We remark that the slopes and intercepts of the linear fits obtained by independently fitting the data for IP shock events (blue) and ${ }^{3} \mathrm{He}$-rich SEP events (green) are well within the respective $1 \sigma$ error limits of the fit parameters for the three types of events. The figure shows the following: (1) Enhancements in the $\mathrm{S} / \mathrm{O}$ and $\mathrm{Ca} / \mathrm{O}$ ratios are accompanied by simultaneous enhancements in the $\mathrm{Fe} / \mathrm{C}$ ratio. (2) The fits to the data provide an excellent representation of the event-to-event variations and the enhancement pattern of the heavyion abundances in LSEP events. (3) For each element, the powerlaw dependence in LSEP events provides remarkably good fits to the corresponding event-to-event variations seen in IP shock events and in ${ }^{3} \mathrm{He}$-rich SEP events.

\subsection{M/Q-dependent Fractionation}

We made plots similar to Figure 14 and obtained the slopes for the rest of the heavy-ion abundances in LSEP events. These slopes are plotted versus $M / Q$ in Figure 15. As in Figure 13, the charge states are taken as the mean ionization states measured in gradual SEP events. The ${ }^{3} \mathrm{He}$ is assumed to have a charge state of $2+$. The scatter of the data about the linear fit, the value of the correlation coefficient $r=0.97$ along with its statistical significance $\left(p \sim 3.4 \times 10^{-4} \%\right)$ indicate that the heavy-ion abundances in LSEP events in our survey are indeed fractionated systematically according to the $M / Q$ ratio (see also Breneman \& Stone 1985). Note that ${ }^{3} \mathrm{He}$ is excluded from the fit and the regression analysis.

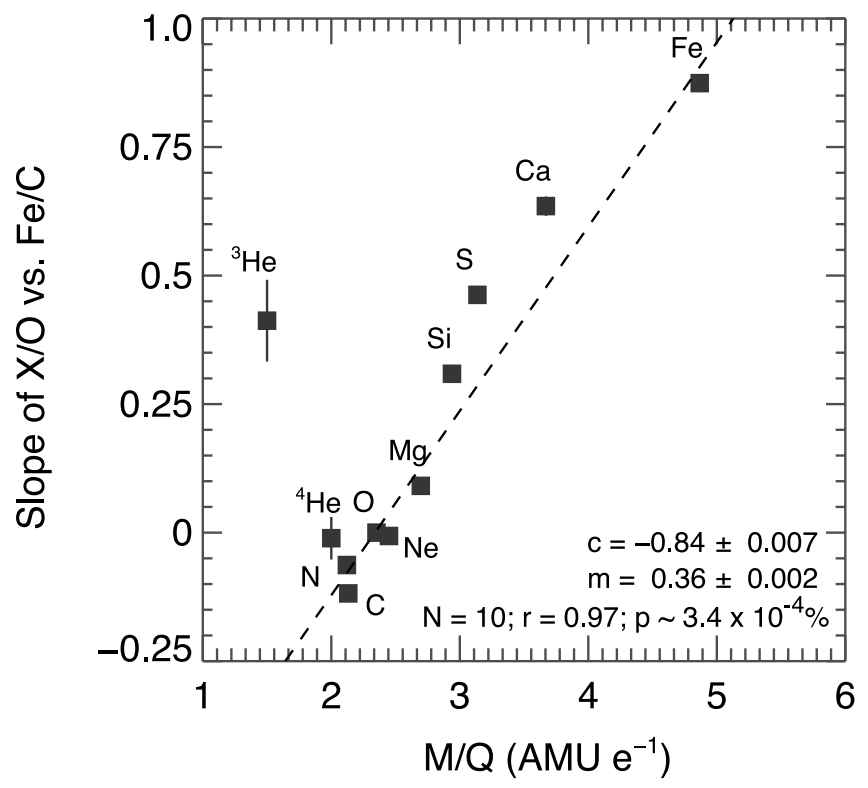

FIG. 15.- Slopes from fits to the elemental abundances $X / \mathrm{O}$ vs. $\mathrm{Fe} / \mathrm{C}$ for LSEP events (e.g., from Figs. $14 a$ and 14b) plotted vs. particle mass/charge $\left(M / Q: \mathrm{amu} e^{-1}\right)$. [See the electronic edition of the Journal for a color version of this figure.] 


\section{SUMMARY OF OBSERVATIONS}

The main results of our survey of the abundances of $\mathrm{He}-\mathrm{Fe}$ in 64 LSEP events of cycle 23 may be summarized as follows.

1. The average abundances derived from all the events are roughly similar to prior surveys at higher energies; however, normalizing to $\mathrm{O}$, we see larger abundances of heavier elements with an $\mathrm{Fe} / \mathrm{O}$ ratio about a factor of 3 greater than higher energy surveys. Elements with similar masses such as $\mathrm{C}$ and $\mathrm{O}$ show no energy dependence in their abundance, while $\mathrm{Fe} / \mathrm{O}$ increases toward lower energies. The $\mathrm{Fe} / \mathrm{O}$ ratio in 55 of the $64 \mathrm{LSEP}$ events in our survey is enhanced when compared with the average slow solar wind value. The $\mathrm{Fe} / \mathrm{O}$ ratio decreases with increasing energy between $\sim 0.1$ and $60 \mathrm{MeV}$ nucleon $^{-1}$ for most of the LSEP events in our survey. Slightly less than half of the events have finite ${ }^{3} \mathrm{He}$ abundances, ranging from $\sim 2$ to $\sim 150$ times the solar wind value. Compared with fast or slow solar wind abundances, the LSEP abundances exhibit unsystematic differences, i.e., they are not ordered by simple physical parameters such as mass or $M / Q$ ratio.

2. The heavy-ion abundances show considerable differences from one event to the next, with ratios such as $\mathrm{Fe} / \mathrm{O}$ varying by more than an order of magnitude. These abundance variations are not correlated with dynamical properties of the associated solar event such as CME speed or flare longitude; they are not correlated with the event fluence; they do not correlate with the solar activity cycle (although the fraction of events with finite ${ }^{3} \mathrm{He}$ does correlate with solar cycle). In addition, variations in $\mathrm{Fe} / \mathrm{O}$ do not correlate with the $\mathrm{Fe} / \mathrm{O}$ measured in the ambient period prior to the particle event onset.

3. Event-to-event abundance variations are reasonably well ordered by mass or mass per charge, so if the relative abundances for each event are plotted versus a reference ratio from the same event (i.e., $\mathrm{S} / \mathrm{O}$ vs. $\mathrm{Fe} / \mathrm{C}$ ), the distribution of points from the events in our survey can be fitted by a power law. Remarkably, the power-law dependence we derive from our LSEP survey also fits event-to-event variations seen in independent surveys of IP shock-associated events and impulsive ${ }^{3} \mathrm{He}$-rich solar flare events.

\section{DISCUSSION}

\subsection{Previous Surveys}

Surveys of SEP abundances carried out in the 1980s and 1990s derived average element abundances and measured the considerable variations observed from event to event. Since the ionization states measured in several gradual SEP events were indicative of typical coronal temperatures, these studies compared the SEP abundances to the corona and attributed differences between coronal and SEP abundances to acceleration and transport effects (e.g., Mason et al. 1980; Cook et al. 1984; Breneman \& Stone 1985; McGuire et al. 1986; Reames 1995a). In many studies, these derived SEP average abundances were then used to derive solar photospheric abundances by application of a correction factor based on the ion's first ionization potential. The modest (less than a factor of $\sim 4$ ) differences between the SEP abundances and the coronal or photospheric abundances were commonly assumed to arise due to errors in the coronal or photospheric abundance determinations.

Since these earlier studies, four major observational advances have taken place, making it possible to discuss the SEP abundances and their variations with fewer assumptions. First, evidence accumulated that in many or most LSEP events, the acceleration took place in association with large-scale shocks that accelerated particles at coronal heights of several solar radii or more (Mason et al. 1984; Cane et al. 1986, 1988; Gosling 1993; Kahler 1994; Reames 1995b). The accelerated material was therefore presumably the solar wind, which dominates the thermal ion abundances in the acceleration region. The second key advance was the development of instruments capable of directly measuring the solar wind ion composition and its variations (Gloeckler et al. 1992; Geiss et al. 1996), which provided definitive abundances that could be compared with the SEPs. Third, improved ionization state measurements over a broader energy range found energy dependence of the charge states, giving evidence for stripping of some accelerated material (e.g., Oetliker et al. 1997; Möbius et al. 2000; Klecker et al. 2006). Fourth, advanced instruments on $A C E$ identified many SEP elements over a broad energy range, making it possible to quantify the affects of particle energy spectra on the average abundances.

\subsection{Results of This Survey}

Our present survey of heavy- ion abundances between $\sim 0.1$ and $10 \mathrm{MeV}$ nucleon ${ }^{-1}$ shows complex differences between the average SEP abundances and the solar wind (Fig. 12) and extremely large ( 2 orders of magnitude) variations from event to event in ratios such as Fe/C (Fig. 14). Similar features are seen also in the SEP population at energies an order of magnitude higher, making it unlikely that the spectral effects are playing a critical role (Mewaldt et al. 2006).

We now discuss whether acceleration and transport effects could explain the large enhancements in the $\mathrm{Fe} / \mathrm{O}$ ratio and the systematic $M / Q$-dependent enhancements observed during the LSEP events surveyed here. The fact that the $\mathrm{Fe} / \mathrm{O}$ ratio decreases with increasing energy (see Fig. 5) indicates that the acceleration processes occurring in most LSEP events are very similar to those operating in CME-driven IP shock events near $1 \mathrm{AU}$ (e.g., Desai et al. 2003; 2004), where ions with higher $M / Q$ ratios are accelerated less efficiently. Shock acceleration can indeed produce large abundance variations, but these are generally observed well above the ULEIS energy range. They are caused by spectral features when heavy-ion spectra fall off more rapidly than lighter species, leading to large changes at high energies (Mazur et al. 1992; Tylka et al. 2001; Cohen et al. 2005; Mewaldt et al. 2005a). This is consistent with the results shown in Figure 5 where the $\mathrm{Fe} / \mathrm{O}$ ratio does indeed fall off toward higher energy, but the effects are most pronounced only when we enter the SIS energy range above $\sim 10 \mathrm{MeV}$ nucleon $^{-1}$. For our low-energy data Figures 4 and 5 show only modest energy variation over our entire energy range, and so we can rule out acceleration as the cause of the large event-to-event variations. Furthermore, such mechanisms can only deplete the abundances of heavier ions like Fe with respect to O (e.g., see Ellison \& Ramaty 1985; Lee 2005; Channok et al. 2005) and therefore cannot account for the systematic $M / Q$-dependent enhancements in the heavy-ion abundances that span more than an order of magnitude when compared with the solar wind values (see Figs. 5 and 14).

Transport effects also do not appear to be capable of affecting our average abundances significantly since they affect the abundances most strongly during the event onsets when intensities are rising (e.g., Mason et al. 1983; Tylka et al. 1999). Since relatively few particles arrive during the rise phase, the onset-time abundance variations do not have a large effect on the eventintegrated values. This is supported by the fact that the eventintegrated $\mathrm{Fe} / \mathrm{O}$ values correlated closely with the $1 \mathrm{hr}$ averages, which give greater weight to the onset phase (see $\S 2.6$ ). 
Another possibility is that more complex processes involving both particle acceleration and release from the shock can result in systematic changes in the event-averaged abundances compared to the seed population. For example, Li et al. (2005) carried out calculations of shock acceleration of SEPs with a model that includes many features believed to operate in these events and showed that at low energies, event-averaged enhancements of $\mathrm{Fe} / \mathrm{O}$ of a factor of $\sim 2$ can take place (accompanied by decreased $\mathrm{Fe} / \mathrm{O}$ at higher energies). Li et al. showed only one example of a strong shock event that might be appropriate for the LSEP events studied here; however, it is not clear whether the increased $\mathrm{Fe} / \mathrm{O}$ they report is a general result that would be expected to be present in almost all cases. If it is, then it might explain the average increase of $\mathrm{Fe} / \mathrm{O}$ we see at lower energies (Fig. 4) and some or all of the $\mathrm{Fe} / \mathrm{O}$ enhancement compared with the solar wind (Fig. 12). However, the abundance enhancements in the Li et al. model are ordered by particle $M / Q$ ratio, so they would not reproduce the features of Figure 12 where there are, e.g., different enhancements for elements of the same $M / Q$ ratio. In addition, in the Li et al. model, the variations in $\mathrm{Fe} / \mathrm{O}$ are much smaller than the event-toevent $\mathrm{Fe} / \mathrm{O}$ differences we observe (in Figs. 5 and 14), and so these larger enhancements would require another mechanism.

\subsection{Is Solar Wind the Dominant Source Population for LSEP Events?}

The $\sim 1 \mathrm{MeV}$ nucleon ${ }^{-1}{ }^{3} \mathrm{He} /{ }^{4} \mathrm{He}$ ratio is enhanced between factors of $\sim 2$ and 150 over the corresponding slow solar wind value in about $46 \%$ of LSEP events in our survey, while about $30 \%$ of the events show enhancements of a factor of $\sim 4-150$. Enrichments in the ${ }^{3} \mathrm{He}$ abundance over the solar wind value are observed frequently in LSEP events (e.g., Mason et al. 1999a; Cohen et al. 2000; Wiedenbeck et al. 2000; Torsti et al. 2002; Kocharov \& Torsti 2003) and in IP shock events (Desai et al. 2001, 2003). This is clear evidence for the presence of suprathermal ${ }^{3} \mathrm{He}$-rich flare material in the seed population since no alternative mechanism has been identified in which shocks could cause such large ${ }^{3} \mathrm{He}$ enhancements compared to the solar wind.

The only known source for significant amounts of ${ }^{3} \mathrm{He}$ is impulsive SEP events, and therefore if ${ }^{3} \mathrm{He}$ is present in the seed population, other heavy elements from the impulsive SEP population should be present also. Mewaldt et al. (2002, 2003, 2006) investigated whether there is enough impulsive SEP material in the IP medium to account for Fe enrichments in LSEP events and concluded on the basis of simple models that there is a shortfall by a factor of $\sim 10-20$. Thus, the current picture is that although impulsive SEP material is definitely present in the seed population for LSEP events, it cannot account for the LSEP abundances in a simple way.

What about the SEP events in our survey that have no apparent enhancement of ${ }^{3} \mathrm{He}$ or Fe? Could at least these events be accelerating a seed population that is simply related to solar wind abundances? We explored this in Figure $12 b$, which shows average abundances for nine events with upper limits for the ${ }^{3} \mathrm{He} /{ }^{4} \mathrm{He}$ ratio and with $\mathrm{Fe} / \mathrm{O}$ ratios similar to the average slow solar wind value. Of all the events in our survey, these events would seem to be the most likely to have accelerated material with composition like the solar wind. However, Figure $12 b$ shows that the average abundances in these events when normalized to the corresponding solar wind values are uncorrelated with the $M / Q$ ratio and therefore are not related to solar wind elemental abundances in any simple manner. It is important to note that a similar conclusion was deduced for SEPs above $\sim 5 \mathrm{MeV}$ nucleon $^{-1}$ by Mewaldt et al. (2002), thus ruling out SEP spectral effects as the cause of these differences.

\subsection{A Simple Model for Deducing the Seed Population for Heavy Ions in LSEP Events}

The above arguments point to the suprathermal ions as the source population for the SEP events in this survey and show that the composition of the suprathermals is not related in a simple manner to the solar wind abundances. Of course it is desirable to compare the average SEP abundances with the presumed source, i.e., suprathermal ions upstream of the shock accelerating the particles. We have already seen (Fig. 9) that sampled at $A C E$, these populations do not reveal any significant correlation, even though a similar comparison found highly significant correlations of this kind for particles associated with IP shock passages at $1 \mathrm{AU}$ (Desai et al. 2003, 2004). We believe that this poor correlation may be due to the fact that the SEPs are accelerated closer to the Sun, and in particular, the magnetic connection to the Sun results in $A C E$ sampling acceleration during most of the events that took place on magnetic field lines connected to the western solar hemisphere. Solar wind from that location crosses 1 AU well in front of the Earth and is not sampled by $A C E$. (This is not the case for the IP shocks, since the acceleration is closer to 1 AU.) Future measurements by $A C E$ along with the STEREO spacecraft could provide the needed observational data to test this correlation.

In addition to this sampling problem, we cannot even make average comparisons between our inferred SEP source and the suprathermal ion populations, since there are only scattered measurements of the suprathermal ion composition. However, since shock acceleration typically depletes heavier mass ions, as seen here in Figure 5 and in surveys of IP shocks (e.g., Klecker et al. 1981; Desai et al. 2003, 2004), presumably the source population is even more abundant in heavy ions than the SEP average abundances presented here. Desai et al. (2003) found that the heavy-ion abundances in IP shocks were related to those measured in the ambient suprathermal population measured prior to the arrival of the shocks at $1 \mathrm{AU}$ according to the simple relation $\log \left(\Gamma_{X}\right)=c+m\left[\left(M_{X} Q_{\mathrm{O}}\right) /\left(Q_{X} M_{\mathrm{O}}\right)\right]$, where $c=0.59 \pm$ $0.06, m=-0.62 \pm 0.06$, and $M_{X} / Q_{X}$ is the $M / Q$ ratio of element $X$. Here $\Gamma_{X}=(X / \mathrm{O})_{S} /(X / \mathrm{O})_{A}$ is the enhancement or depletion of the $X / \mathrm{O}$ ratio in IP shocks, $(X / \mathrm{O})_{S}$, relative to that in the suprathermal population, $(X / \mathrm{O})_{A}$. Thus, if we merely apply this function (see Fig. 12 of Desai et al. 2003) to the average LSEP abundances given in Table 3, we can infer average abundances for the "source" population. In applying this function to our data we are assuming a simple, linear mixing with no energy dependence, and so the result should be seen as illustrative only.

The inferred "source" SEP population is compared in Figure 16 with spectroscopic measurements of the $\sim 1.4$ MK quiet corona (Feldman \& Widing 2003), average values measured in ${ }^{3} \mathrm{He}$-rich SEP events (Mason et al. 2004), and LSEP events as a function of atomic mass. It can be seen that the heavy ions in this source population are greatly enhanced over coronal values. If we assume that the inferred LSEP heavy-ion source abundances are a linear mixture of quiet coronal and heavy-ion-enriched impulsive material, then about $75 \%$ of the heavy ions would come from the impulsive material. Of course, other possibilities exist, including that the heavy-ion enrichment in the LSEP source is due to a mechanism different from that operating in impulsive SEP events.

\subsection{Origin of the Suprathermal Seed Population for SEP Events}

The above discussion outlines the evidence that LSEP events originate from suprathermal material, whose average abundance 


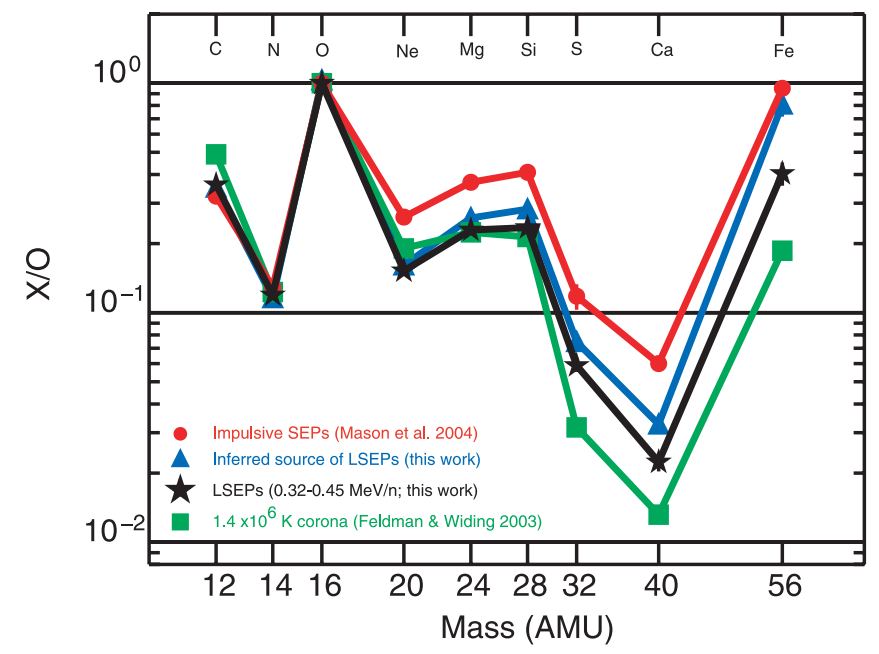

FIG. 16.-Elemental abundances of LSEP events (stars), compared with average abundances in ${ }^{3} \mathrm{He}$-rich SEP events (circles) from Mason et al. (2004), the inferred source abundances for LSEP events (triangles), and spectroscopic abundances of the quiet $\sim 1.4 \times 10^{6} \mathrm{~K}$ solar corona (squares) from Feldman \& Widing (2003).

is significantly enriched in Fe and the heavier ions compared to the solar wind. What is the source of this material? Several authors have pointed out that there are many contributors to the suprathermal ion population including remnants of impulsive and gradual SEP events, CIR particles, and IP shocks, as well as more localized contributors such as planetary magnetospheres and comets (e.g., Gosling et al. 1981; Tsurutani \& Lin 1985; Mason 2000; Mewaldt et al. 2006). For CME-driven shocks within a few solar radii, most of these sources should be unimportant, leaving only gradual and impulsive SEPs and the heated coronal or solar wind material as potential sources.

Our discussion so far has treated the solar wind as a fixed average quantity, while of course variations in the elemental composition are well known (e.g., von Steiger et al. 1995a). However, variations in the solar wind ion composition tend to be of order a factor of $\sim 4$ or less (e.g., Wurz et al. 2000) and so could be important for the average enrichment of Fe we see in SEPs, but are much less than the observed event-to-event variations that cover more than an order of magnitude. It could be that a more complex process could still put heated solar wind into the suprathermal ion pool in a way that could lead to much larger heavy-ion enhancements. For example, it has been long known that solar wind heavy-ion temperatures scale proportionally to their mass (Ogilvie et al. 1980; Schmidt et al. 1980; von Steiger et al. 1995b), and conceivably this could lead to a situation in which the higher mass ions were heated to proportionally higher energies in the suprathermal region, thereby enabling more efficient acceleration by some additional mechanism. Although conceptually this complex scenario could result in the very large event-to-event variations that are observed, it is not clear if it could actually explain the simultaneous depletions and the enrichments of ions of similar $M$ and $M / Q$ (e.g., O vs. C, N, Ne, and $\mathrm{Mg}$ ).

Another possibility is that impulsive flare material, which is rich in heavy ions, is even more widely present near the Sun than generally thought. This possibility is raised by the fact that ${ }^{3} \mathrm{He}$ enrichments are seen in the IP medium for the majority of the time during solar active periods (Wiedenbeck et al. 2003), perhaps indicating that a quasi-continuous mechanism rather than short-lived episodes produces this heavy-ion-rich material. If impulsive material is routinely present near the Sun, then this might provide the seed population accelerated by the large shocks associated with SEP events. In this case, it would make it relatively easy to understand why the event-to-event variations in the LSEP events surveyed here are remarkably similar to those seen in impulsive events but offset by a factor (Fig. 14). On the other hand, if the LSEP heavy-ion enrichment is due to kinetic properties of the solar wind as suggested above, then it seems that the similarity of the event-to-event variations seen in LSEP events and in IP shock events when compared with those seen in impulsive SEP events (Fig. 14) is just a coincidence.

No doubt other possibilities exist; for example, the heavy-ionenriched suprathermal seed population could originate from flares that typically accompany the CMEs (e.g., Li \& Zank 2005 and references therein). The mere existence of numerous possibilities clearly illustrates that comprehensive new observations of the suprathermal energy regime are needed to remove the current ambiguities. In any case, it is clear that the results presented here cannot be reconciled with the currently held viewpoint that large gradual SEP events are produced by the acceleration of ambient coronal or solar wind material at CME-driven shocks near the Sun and in IP space. Instead, it appears that a comprehensive model for LSEP events must include the routine production of heavy-ion-enriched suprathermal seed population as a critical precursor to the CME shock acceleration process to account for the new observations reported here.

We are grateful to the members of the Space Physics Group, University of Maryland and the Johns Hopkins Applied Physics Laboratory (JHU/APL) for the construction of the ULEIS instrument. Work at the University of Maryland was supported by NASA contract NAS5-30927 and NASA grant PC 251428; work at Southwest Research Institute was partially supported by NASA grant NNG05GQ94G and NSF grant ATM 05-55878; work at JHU/APL was supported by NASA grant NNG04GJ51G. Work at the California Institute of Technology was supported by NASA grants NAG5-12929 and NNG05GQ94G and NSF grant ATM 04-54428. We acknowledge the use of the lists of Solar Proton Events Affecting the Earth Environment provided by the NOAA Space Environment Services Center at http://solar.sec .noaa.gov/ftpdir/SPE.txt and that of CMEs provided by the $\mathrm{SOHO}$ LASCO team at http://cdaw.gsfc.nasa.gov/CME_list.
Bodmer, R., et al. 1995, Space Sci Rev., 72, 61

Bone, N. M. 1992, S\&T, 84, 524

Breneman, H. H., \& Stone, E. C. 1985, ApJ, 299, L57

Bryant, D. A., et al. 1962, J. Geophys. Res., 67, 4983

Cane, H. V., McGuire, R. E., \& von Rosenvinge, T. T. 1986, ApJ, 301, 448

Cane, H. V., Reames, D. V., \& von Rosenvinge, T. T. 1988, J. Geophys. Res., 93, 9555

Cane, H. V., et al. 2003, Geophys. Res. Lett., 30, SEP 5-1 2006, J. Geophys. Res., 111, A06S90

\section{REFERENCES}

Channok, C., et al. 2005, ApJ, 633, L53

Cliver, E. W. 2000, in AIP Conf. Proc. 528, Acceleration and Transport of Energetic Particles Observed in the Heliosphere, ed. R. A. Mewaldt et al. (New York: AIP), 21

Cliver, E. W., et al. 1982, ApJ, 260, 362

Cohen, C. M. S., et al. 1999, Geophys. Res. Lett., 26, 149 2000, in AIP Conf. Proc. 528, Acceleration and Transport of Energetic Particles Observed in the Heliosphere, ed. R. A. Mewaldt et al. (New York: AIP), 55 
Cohen, C. M. S., et al. 2003, Adv. Space Res., 32, 2649 2005, J. Geophys. Res., 110, A09S16

Cook, W. R., Stone, E. C., \& Vogt, R. E. 1984, ApJ, 279, 827

Desai, M. I., et al. 2001, ApJ, 553, L89

2003, ApJ, 588, 1149

2004, ApJ, 611, 1156

Dietrich, W. F. 1973, ApJ, 180, 955

Dryer, M. 1987, Sol. Phys., 114, 407

Ellison, D. C., \& Ramaty, R. 1985, ApJ, 298, 400

Feldman, U., \& Widing, K. G. 2003, Space Sci. Rev., 107, 665

Forbush, S. E. 1946, Phys. Rev., 70, 771

Forman, M. A., \& Webb, G. 1985, in Collisionless Shocks in the Heliosphere: A Tutorial Review, ed. R. G. Stone \& B. T. Tsurutani (Geophys. Monogr. 35; Washington: AGU), 91

Garrard, T. L., Stone, E. C., \& Vogt, R. E. 1973, in High Energy Phenomena on the Sun, ed. R. Ramaty \& R. G. Stone (NASA SP-342; Greenbelt: NASA), 341

Geiss, J., Gloeckler, G., \& von Steiger, R. 1996, Space Sci. Rev., 78, 43

Gloeckler, G. 2003, in AIP Conf. Proc. 679, Solar Wind Ten, ed. M. Velli et al. (New York: AIP), 583

Gloeckler, G., \& Geiss, J. 1998, Space Sci. Rev., 84, 275

Gloeckler, G., et al. 1976, ApJ, 209, L93 . 1992, A\&AS, 92, 267

Gopalswamy, N., et al. 2005a, in Proc. 29th Int. Cosmic Ray Conf. (Pune), 1, 169 . 2005b, J. Geophys. Res., 110, A12S07

Gosling, J. T. 1993, J. Geophys. Res., 98, 18937

Gosling, J. T., et al. 1981, J. Geophys. Res., 86, 547

Ho, G. C., et al. 2000, Geophys. Res. Lett., 27, 309

Hovestadt, D., et al. 1981, Adv. Space Res., 1, 61

Kahler, S. 1994, ApJ, 428, 837

. 2005, ApJ, 628, 1014

Kahler, S., W., Hildner, E., \& Van Hollebeke, M. A. I. 1978, Sol. Phys., 57, 429

Kahler, S. W., et al. 1984, J. Geophys. Res., 89, 9683

Kennel, C. F., et al. 1986, J. Geophys. Res., 91, 11917

Klecker, B., et al. 1981, ApJ, 251, 393

1984, ApJ, 281, 458

1999, in Proc. 26th Int. Cosmic Ray Conf. (Salt Lake City), 6, 83

2000, in AIP Conf. Proc. 528, Acceleration and Transport of Energetic

Particles Observed in the Heliosphere, ed. R. A. Mewaldt et al. (New York: AIP), 135

. 2006, Adv. Space Res., in press

Kocharov, G. E. 1983, Proc, 18th Int. Cosmic Ray Conf. (Bangalore), 12, 235

Kocharov, L., \& Torsti, J. 2003, ApJ, 586, 1430

Labrador, A. W., et al. 2003, Proc. 28th Int. Cosmic Ray Conf. (Tsukuba), 6, 3269

Lee, M. A. 1983, J. Geophys. Res., 88, 6109 2005, ApJS, 158, 38

Leske, R. A., et al. 2003, in AIP Conf. Proc. 679, Solar Wind Ten, ed. M. Velli et al. (New York: AIP), 616

Li, G., \& Zank, G. P. 2005, Geophys. Res. Lett., 32, L02101

Li, G., Zank, G. P., \& Rice, W. K. M. 2005, J. Geophys. Res., 110, A06104

Lin, R. P. 1970, Sol. Phys., 12, 266

Lodders, K. 2003, ApJ, 591, 1220

Luhn, A., et al. 1984, Adv. Space Res., 4, 161 1987, ApJ, 317, 951

Mason, G. M. 2000, in AIP Conf. Proc. 528, Acceleration and Transport of Energetic Particles Observed in the Heliosphere, ed. R. A. Mewaldt et al. (New York: AIP), 234

Mason, G. M., Gloeckler, G., \& Hovestadt, D. 1983, ApJ, 267, 844

Mason, G. M., Mazur, J. E., \& Dwyer, J. R. 1999a, ApJ, 525, L133

Mason, G. M., et al. 1980, ApJ, 239, 1070 1984, ApJ, 280, 902
Mason, G. M., et al. 1997, ApJ, 486, L149

1998, Space Sci. Rev., 86, 409

1999b, Geophys. Res. Lett., 26, 141

2002, ApJ, 574, 1039

2004, ApJ, 606, 555

Mazur, J. E., et al. 1992, ApJ, 401, 398

1993, ApJ, 404, 810

1999, Geophys. Res. Lett., 26, 173

2000, ApJ, 532, L79

McGuire, R. E., von Rosenvinge, T. T., \& McDonald, F. B. 1986, ApJ, 301, 938

Mewaldt, R. A., Cohen, C. M. S. C., \& Mason, G. M. 2006, in Solar Eruptions and Energetic Particles, ed. N. Gopalswamy, R. A. Mewaldt, \& J. Torsti (Washington: AGU), in press

Mewaldt, R. A., et al. 2002, Adv. Space Res., 30, 79 2003, Proc. 28th Int. Cosmic Ray Conf. (Tsukuba), 6, 3229 2005a, in AIP Conf. Proc. 781, The Physics of Collisionless Shocks,

ed. G. Li, G. P. Zank, \& C. T. Russell (New York: AIP), 227 2005b, J. Geophys. Res., 110, A09S18

Meyer, J. P. 1985, ApJS, 57, 151

Meyer, P., Parker, E. N., \& Simpson, J. A. 1956, Phys. Rev., 104, 768

Möbius, E., et al. 1999, Geophys. Res. Lett., 26, 145 . 2000, in AIP Conf. Proc. 528, Acceleration and Transport of Energetic

Particles Observed in the Heliosphere, ed. R. A. Mewaldt et al. (New York: AIP), 131

Oetliker, M., et al. 1997, ApJ, 477, 495

Ogilvie, K. W., et al. 1980, J. Geophys. Res., 85, 6021

Popecki, M. A., et al. 2002, Adv. Space Res., 30, 33

Reames, D. V. 1988, ApJ, 330, L71

- 1995a, Adv. Space Res., 15(7), 41

. 1995b, Rev. Geophys., 33, 585 1999, Space Sci. Rev., 90, 413

Rust, D. M. 1987, in The Solar Wind and the Earth, ed. S.-I. Akasofu \& Y. Kamide (Dordrecht: Reidel), 2

Schmidt, W. K. H., et al. 1980, Geophys. Res. Lett., 7, 697

Sheeley, N. R., Jr. 1996, ApJ, 469, 423

Slocum, P. L., et al. 2003, ApJ, 594, 592

Sollitt, L. S., et al. 2003, Proc. 28th Int. Cosmic Ray Conf. (Tsukuba), 6, 3295

Stone, E. C., et al. 1998a, Space Sci. Rev., 86, 1 1998b, Space Sci. Rev., 86, 357

Tan, L. C., et al. 1989, ApJ, 345, 572

Torsti, J., et al. 2002, ApJ, 573, L59

Tsurutani, B. T., \& Lin, R. P. 1985, J. Geophys. Res., 90, 1

Tylka, A. J., Reames, D. V., \& Ng, C. K. 1999, Geophys. Res. Lett., 26, 2141

Tylka, A. J., et al. 2001, ApJ, 558, L59 2005, ApJ, 625, 474

Van Hollebeke, M. A. I., McDonald, F. B., \& Meyer, J. P. 1990, ApJS, 73, 285 von Steiger, R., Geiss, J., \& Gloeckler, G. 1997, in Cosmic Winds and the Heliosphere, ed. J. R. Jokipii, C. P. Sonnett, \& M. S. Giampapa (Tucson: Univ. Arizona Press), 581

von Steiger, R., et al. 1995a, Adv. Space Res., 15(7), 3 1995b, Space Sci. Rev., 72, 71 2000, J. Geophys. Res., 105, 27217

Wiedenbeck, M. E., et al. 2000, in AIP Conf. Proc. 528, Acceleration and Transport of Energetic Particles Observed in the Heliosphere, ed. R. A. Mewaldt et al. (New York: AIP), 107 . 2003, in AIP Conf. Proc. 679, Solar Wind Ten, ed. M. Velli, R. Bruno, \& F. Malara, (New York: AIP), 652

Wild, J. P., Smerd, S. F., \& Weiss, A. A. 1963, ARA\&A, 1, 291

Wurz, P., Bochsler, P., \& Lee, M. A. 2000, J. Geophys. Res., 105, 27239

Wurz, P., et al. 2003, ApJ, 583, 489 